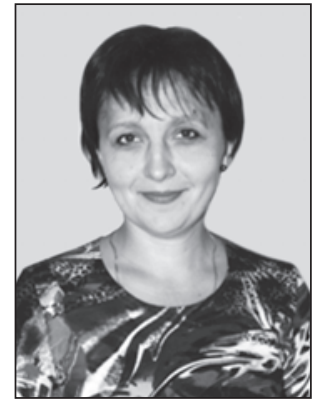

ГРИГОРЬЕВА Н.B.

Институт геронтологии им. Д.Ф. Чеботарева НАМН Украины

Украинский научно-медицинский центр проблем остеопороза, г. Киев

\title{
ГОРМОНАЛЬНАЯ КОНТРАЦЕПЦИЯ И КОСТНАЯ ТКАНЬ
}

Гормональная контрацепция является высокоэффективным и широкодоступным методом предупреждения беременности путем использования синтетических аналогов женских половых гормонов. Кроме того, в настоящее время гормональные контрацептивы (ГК) широко используют для лечения многих заболеваний, связанных с нарушениями синтеза и метаболизма половых гормонов. Надежность и безопасность использования ГК остаются наиболее важными задачами при их использовании (Wiegratz I., Thaler C.J., 2011; Lee J.K., Parisi S.M., Akers A.Y. et al., 2011; http:// www.who.int/reproductivehealth/publications/family planning/9789241563888/en).

В последнее время ведутся активные дискуссии по поводу возможного влияния ГК на состояние костной ткани. С одной стороны, учитывая существующие положительные эффекты заместительной эстроген-гестагенной терапии в предупреждении потери костной ткани и лечении постменопаузального остеопороза и экстраполируя эти данные на применение ГК, обсуждаются положительные эффекты последних в поддержании и улучшении структурно-функционального состояния костной ткани. С другой стороны, существуют данные, что некоторые ГК могут оказывать отрицательное влияние на темпы потери костной ткани, особенно у женщин в пре- и перименопаузальном периоде.

Цель настоящей статьи - проанализировать существуюшие данные относительно влияния гормональных контрацептивов различных групп на структурно-функциональное состояние костной ткани у женщин в репродуктивном и пременопаузальном периодах жизни, а также при нарушениях оварио-менструального цикла.

\section{Классификация гормональных контрацептивов и механизмы их действия}

В настоящее время существуют две формы гормональной контрацепции - оральная (таблетированная) и парентеральная (импланты, инъекции, внутриматочные гормональные системы и влагалищные кольца) (Kuhl H., 2005; Guilbert E., Black A., Dunn S. et al.,
2008; Wiegratz I., Thaler C.J., 2011; Lee J.K., Parisi S.M., Akers A.Y. et al., 2011).

Оральные контрацептивы (ОК) появились в начале 60-х годов XX века и до сих пор остаются одним из самых надежных и удобных методов контроля рождаемости. По данным Всемирной организации здравоохранения (ВО3), в настоящее время около 100 млн женщин принимают гормональные контрацептивы (http:// www.who.int/reproductivehealth/publications/family_planning/9789241563888/en). По статистическим данным Canadian National Health Population Survey, 18 \% канадских женщин в возрасте 15-49 лет используют именно оральные методы контрацепции (Wilkins K. et al., 2001).

Эстрогенным компонентом оральных контрацептивов является этинилэстрадиол (ЭЭ), гестагенным различные синтетические гестагены (прогестины). Парентеральные формы ГК содержат либо только гестагенный компонент, либо комбинацию эстрогенов и прогестагенов (влагалищные кольца). Преимущество в использовании имеют оральные формы контрацепции, которые являются комбинированными (эстроген-гестагенными), хотя в настоящее время существуют возрастные и этнические особенности использования различных методов контрацепции (Wiegratz I., Thaler C.J., 2011; Lee J.K., Parisi S.M., Akers A.Y. et al., 2011).

В зависимости от схемы комбинации эстрогена и гестагена выделяют следующие группы комбинированных оральных контрацептивов (КОК): монофазные, содержащие неизменяющуюся дозу эстрогена и гестагена в каждой таблетке; двухфазные, которые содержат одинаковую дозу эстрогена во всех таблетках, однако доза гестагена варьирует (сначала минимальная, а потом более высокая), и трехфазные КОК, в которых содержание гормонов в таблетке меняется согласно фазам менструального цикла (первые таблетки содержат низкие дозы эстрогена и гестагена, в последующих они повышаются, а в последних таблетках доза эстрогена воз-

(C) Григорьева Н.В., 2013

(c) «Боль. Суставы. Позвоночник», 2013

(c) Заславский А.Ю., 2013 
вращается к минимальной, а гестагена повышается еще больше) (Rimsza M.E., 2003) (табл. 1).

С клинической точки зрения также важно деление КОК на группы в зависимости от дозы ЭЭ. Комбинированные оральные контрацептивы подразделяют на высокодозированные (содержат 50 мкг/д ЭЭ); низкодозированные ( $\leq 35$ мкг/д ЭЭ) и микродозированные (15-20 мкг/д ЭЭ). КОК, содержащие более 50 мкг/д ЭЭ, в настоящее время не используются. Высокодозированные КОК (антеовин, овидон, нон-овлон и др.) не должны применяться длительно, их назначают с лечебной целью для увеличения дозы эстрогена. Для гормональной контрацепции в настоящее время применяют низкодозированные (диане- 35 , жанин, минизистон, марвелон, триквилар, тризистон, три-мерси, фемоден, ярина, белара и др.) и микродозированные (логест, мирель, мерсилон, новинет и др.) КОК. В последние десятилетия развитие гормональной контрацепции идет по пути снижения дозы эстрогенов и создания селективных прогестинов.

Этинилэстрадиол - синтетический стероидный эстроген, этинильная группа в молекуле которого замедляет его биологическое разрушение ферментами и усиливает связывание с эстрогенными рецепторами, в связи с чем он обладает более сильными эстрогенными свойствами по сравнению с природным эстрадиолом. Ранее в США широко использовался местранол, однако в настоящее время он почти не применяется.

Гестагенный компонент КОК составляют синтетические прогестины, которые подразделяют на производные прогестерона или нортестостерона (табл. 2). Синтетические гестагены оказывают гестагенное и антиэстрогенное действие, необходимое для их контрацептивного эффекта, однако некоторые из них также обладают андрогенным, минерало- и глюкокортикоидным действием. Для некоторых прогестинов харак- терны «антигормональные» (антиандрогенный и антиминералокортикоидный) эффекты.

Группа производных 19-нортестостерона - активные гестагены, обладающие остаточным андрогенным эффектом. Первое поколение - наиболее старые гестагены (норэтистерон, этинодиола ацетат, линестренол) с относительно высокой андрогенной активностью в настоящее время в составе КОК почти не используются Гестагены второго поколения (норгестрел, левоноргестрел) обладают более выраженной гестагенной активностью и менее выраженными андрогенными свойствами, чем прогестины первого поколения. Гестагены третьего поколения (гестоден, дезогестрел, норгестимат) характеризуются высокой гестагенной активностью и минимальным (клинически незначимым) андрогенным эффектом, поэтому именно эти гестагены широко используют в современных контрацептивах.

Производные прогестерона более разнообразны по своим фармакологическим эффектам. Среди них есть гестагены как с сильным (ципротерон) и слабым (хлормадинон) антиандрогенным действием, так и со слабым андрогенным эффектом (медроксипрогестерон). В настоящее время синтезировано много различных гестагенов - производных прогестерона, однако большинство из них для контрацепции не используют, потому что они не подавляют овуляцию в клинически приемлемых низких дозах. Кроме того, некоторые производные прогестерона (медроксипрогестерона ацетат, мегестрол и др.) при приеме внутрь разрушаются в желудке желудочным соком, поэтому их используют в основном для инъекционной контрацепции.

Производное спиронолактона дроспиренон обладает выраженными гестагенным и антиминералокортикоидным, а также слабым антиандрогенным эффектами.

Наиболее важным механизмом действия КОК является предохранение от возможной беременности, кото-

Таблица 1. Классификация гормональных контрацептивов

\begin{tabular}{|c|c|}
\hline Комбинированные эстроген-гестагенные контрацептивы & Гестагенные контрацептивы \\
\hline Оральные & Оральные (мини-пили) \\
\hline $\begin{array}{l}\text { Монофазные: логест, марвелон, мерсилон, микрогинон, минизистон, } \\
\text { диане-35, жанин, силест, ярина. } \\
\text { Двухфазные: антеовин, депозистон, секвостат, фемоден. } \\
\text { Трехфазные: триквилар, тризистон, три-мерси }\end{array}$ & $\begin{array}{l}\text { Континуин, микролют, микронор, оврет, фемулен, чарозетта, эксклю- } \\
\text { тон }\end{array}$ \\
\hline Парентеральные & Парентеральные \\
\hline $\begin{array}{l}\text { Влагалищные кольца: новаринг. } \\
\text { Пластыри: ортоевра }\end{array}$ & $\begin{array}{l}\text { Импланты: норплант. } \\
\text { Инъекции: депо-медроксипрогестерона ацетат - ДМПА (депо-про- } \\
\text { вера), норэтистерона энантат (НЭТ-ЭН). } \\
\text { Внутриматочная гормональная система: мирена. } \\
\text { Влагалищные кольца, содержащие левоноргестрел, норэтиндрон } \\
\text { или прогестерон }\end{array}$ \\
\hline
\end{tabular}

Таблица 2. Классификация гестагенов, входяших в состав КОК

\begin{tabular}{|l|l|}
\hline \multicolumn{1}{|c|}{ Прогестины } & \multicolumn{1}{|c|}{ Пример } \\
\hline Производные прогестерона (ретропрогестерон) & Дидрогестерон \\
\hline Производные $17 \alpha$ ОН-прогестерона прегнанового происхождения & $\begin{array}{l}\text { Медроксипрогестерона ацетат, ципротерона ацетат, хлормадино- } \\
\text { на ацетат }\end{array}$ \\
\hline Производные 19-нортестостерона эстранового происхождения & $\begin{array}{l}\text { Норэтистерон, этинодиола ацетат, линестренол, норгестрел, лево- } \\
\text { норгестрел }\end{array}$ \\
\hline Производные 19-нортестостерона гонанового происхождения & Дезогестрел, гестоден, норгестимат, диеногест \\
\hline Производные спиронолактона & Дроспиренон \\
\hline
\end{tabular}


рое осуществляется путем подавления овуляции (блокада выброса рилизинг-гормонов гипоталамуса и гонадотропных гормонов гипофиза, а также непосредственное угнетение фолликулогенеза в яичнике), влияния на шеечную слизь (вызывает ее сгущение, что препятствует проникновению сперматозоидов в полость матки), а также на эндометрий (вызывает его изменения, препятствующие имплантации, отсутствие полноценной его пролиферации, ранней и неполноценной секреторной трансформации) (Rimsza M.E., 2003; Wiegratz I., Thaler C.J., 2011; Lee J.K., Parisi S.M., Akers A.Y., 2011).

Парентеральные методы комбинированной эстроген-гестагенной контрацепции предполагают использование влагалищных колец или пластыря.

Влагалищные колыца - гибкие по своей структуре контрацептивы в форме кольца, которые содержат гормоны в минимальных дозах: либо комбинацию прогестагена (120 мкг) и эстрогена (15 мкг), либо только прогестагены (левоноргестрел, норэтиндрон или прогестерон). Доза стероидов во влагалищном кольце гораздо меньше, чем во многих других ГК, что позволяет нивелировать их отрицательные эффекты на организм женщины.

Половые гормоны из кольца, в отличие от ОК, поступают в организм не через желудочно-кишечный тракт, а напрямую всасываются в кровь, проникая через слизистую оболочку влагалища, что предотвращает их отрицательное влияние на слизистую оболочку желудка. Вагинальные кольца вводятся и удаляются самими женщинами, что, по мнению одних женщин, является преимуществом, а других - определенным недостатком данного метода.

Гормональный пластырь как важный метод парентеральной (трансдермальной) контрацепции содержит комбинацию эстрогена и прогестина. Такой пластырь можно носить на руке, ягодицах и животе в течение недели, с последующей еженедельной заменой. Как правило, при использовании данного метода контрацепции отсутствуют побочные эффекты со стороны желудочно-кишечного тракта в связи с парентеральным введением стероидов. Данный метод контрацепции был одобрен только в 2001 году, и на сегодняшний день существующие исследования доказывают его эффективность, соизмеримую с ОК.

Кроме контрацептивного эффекта данные КОК обладают и другими терапевтическими эффектами, связанными и не связанными с менструальным циклом, в связи с чем в настоящее время существуют следующие лечебные показания к назначению КОК: нарушения менструального цикла и дисфункциональные маточные кровотечения; альгодисменорея; предменструальный синдром; функциональные кисты яичников; овуляторные боли; некоторые формы ановуляции с целью достижения ребаунд-эффекта; олигоили аменорея на фоне хронической ановуляции при синдроме поликистозных яичников и/или гиперандрогении; некоторые формы акне, себореи, андрогензависимой алопеции.

Мини-пили содержат микродозы гестагенов (300500 мкг), что составляет 15-30 \% дозы прогестагена в КОК. В препаратах этой группы представлены различные прогестины, например в континуине и фемулене - этинодиола диацетат, микроноре - норэтистерон, оврете - норгестрел, экслютоне - линестренол. Мини-пили принимают в непрерывном режиме начиная с 1-го дня менструального цикла. Их контрацептивный эффект несколько ниже, чем у КОК, и составляет 0,3-9,6 беременности на 100 женщин-лет. Механизм контрацептивного действия мини-пили, как и КОК, заключается во влиянии на шеечную слизь (уменьшение количества и увеличение вязкости, препятствующие проникновению сперматозоидов), эндометрий (развитие изменений, препятствующих имплантации), гипоталамо-гипофизарную систему (подавление овуляции), миграцию яйцеклетки по маточной трубе (замедление ее движения).

Показаниями к назначению мини-пили являются старший репродуктивный возраст, период лактации (4-6 нед. после родов), наличие противопоказаний к назначению эстрогенов, сахарный диабет, активное курение в возрасте старше 35 лет.

Преимущества назначения мини-пили - низкое содержание прогестагена и отсутствие эстрогена в препарате, что связано с меньшим угнетением гипоталамо-гипофизарной системы, со снижением риска развития заболеваний сердечно-сосудистой системы и цереброваскулярных нарушений, а также с отсутствием влияния на углеводный обмен и систему свертывания крови.

Инъекционные контрацептивы представлены прогестагенами пролонгированного действия, лишенными эстрогенной и андрогенной активности. Преимущества этого метода - высокий контрацептивный эффект (0,5-1,5 беременности на 100 женщин-лет), простота применения, низкая частота метаболических нарушений (из-за отсутствия эстрогенного компонента). Инъекции ДМПА (депо-провера) проводят каждые 12 нед., НЭТ-ЭН - 1 раз в 8 нед. Восстановление фертильности происходит в течение 4-24 мес. после последней инъекции. В последнее время используют инъекционные микросферы норэтиндрона (диаметром 0,06-0,1 мм, инъецируются с интервалом 3 месяца, содержат 75 мг норэтиндрона и выделяют в среднем 0,48 мг гормона в сутки).

Механизм контрацептивного действия инъекционных контрацептивов подобен другим гестагенсодержащим методам гормональной контрацепции и заключается в подавлении овуляции, изменениях физико-химических свойств слизи цервикального канала (повышается ее вязкость и волокнистость), препятствующих пенетрации сперматозоидов, развитии изменений в эндометрии, препятствующих имплантации.

Показания к назначению инъекционных контрацептивов - невозможность регулярного ежедневного приема других гормональных препаратов при желании увеличить интервал между родами, поздний репродуктивный возраст (старше 35 лет), противопоказания к назначению эстрогенов, период лактации (6 нед. после родов), использование в качестве послеабортной контрацепции.

Подкожные имплантаты норплант и норплант-2 представлены гибкими пластиковыми капсулами, ка- 
ждая из которых содержит 35 мг левоноргестрела. Механизм их контрацептивного действия основан на выделении капсулами левоноргестрела с постоянной скоростью (30 мкг/сутки), что обеспечивает их контрацептивный эффект, механизм действия которого подобен другим гормональным контрацептивам, содержащим гестагены. Контрацептивная эффективность норпланта достаточно высока и составляет 0,5-1,5 беременности на 100 женщин-лет. Капсулы имплантируют в подкожно-жировую клетчатку внутренней поверхности предплечья через кожный разрез под местной анестезией. Контрацептивная эффективность норпланта сохраняется в течение 5 лет, норпланта 2-3 лет.

Показания к применению подкожных имплантатов поздний репродуктивный возраст, желание пациентки увеличить интервал между родами, наличие эстрогензависимых побочных реакций в анамнезе, заболевания, препятствующие назначению КОК, миома матки небольших размеров (до 8 нед.), фиброзно-кистозная мастопатия, гиперполименорея, альгоменорея и др.

Преимущества метода - его высокий контрацептивный эффект, возможность использования при заболеваниях, исключающих прием КОК, отсутствие влияния на углеводный и липидный обмены (из-за отсутствия эстрогенов), отсутствие неблагоприятного влияния на сердечно-сосудистую систему, удобство применения, связанное с однократным введением препарата и непрерывным пролонгированным действием.

Внутриматочные контрацептивы, содержащие гестагены, представлены, в частности, внутриматочной системой мирена, стержень которой состоит из левоноргестрелсодержащего (52 мг) контейнера, через мембрану которого непрерывно происходит контролируемая диффузия левоноргестрела по 20 мкг/д. Одним из показаний к назначению мирены, кроме необходимости контрацепции, является идиопатическая меноррагия. Механизм действия внутриматочных систем связан с содержашимся гестагеном. Левоноргестрел, непосредственно поступающий в полость матки, оказывает прямое локальное воздействие на эндометрий, предотвращая пролиферативные изменения и препятствуя имплантации, увеличивает вязкость слизи цервикального канала, а также оказывает незначительное системное действие, проявляющееся угнетением овуляции в некотором числе циклов. Контрацептивный эффект мирены поддерживается в течение 5 лет. Способность к деторождению восстанавливается у $50 \%$ женщин через 6 мес. после удаления контрацептива, у $96 \%$ - через 12 мес.

Положительные неконтрацептивные эффекты гормональных контрацептивов связаны с подавлением овуляции и устранением колебаний уровней половых гормонов в организме женщины, что иногда может стать причиной развития некоторых видов патологии.

В настоящее время показано, что кроме лечения состояний, связанных с менструальным циклом, а именно: дисменореи (регуляция менструального цикла), альгоменореи (устранение или уменьшение болевого синдрома во время менструации или овулятор- ных болей), меноррагии и железодефицитной анемии (уменьшение объема менструальной кровопотери), функциональных кист яичников, предменструального синдрома и перименопаузальных симптомов (табл. 3), КОК снижают частоту раковых заболеваний яичников, эндометрия, толстого кишечника, оказывают лечебное влияние на состояние кожи при акне и гирсутизме, а также эффективны в предупреждении эктопической беременности, доброкачественных заболеваний молочной железы, инфекционных заболеваний органов малого таза (Rimsza M.E., 2001; Gambacciani M., 2006; Wiegratz I., Thaler C.J., 2011). Кроме того, в многочисленных исследованиях продемонстрировано влияние ГК на состояние костной ткани.

Однако в то время, как эффекты эстрогенов и гестагенов на структурно-функциональное состояние костной ткани широко описаны в современной литературе, влияние гормональных контрацептивов на состояние костной ткани продолжает оставаться предметом многочисленных споров. Существуют противоречивые данные относительно влияния ОК на костную ткань, которые различаются в зависимости от возраста, эндокринного статуса женщины, а также дозы и типа ОK.

\section{Влияние гормональных контрацептивов на формирование пика костной массы}

Согласно многочисленным данным (Поворознюк B.B., 1999; Bonjiour J.P. et al., 1994; Kroger H. et al., 1995), важной детерминантой структурно-функционального состояния костной ткани у людей старших

Таблица 3. Преимущества оральных контрацептивов (Rimsza M.E., 2003)

\begin{tabular}{|l|c|}
\hline \multicolumn{1}{|c|}{ Состояния/заболевания } & $\begin{array}{c}\text { Снижение риска у женщин, } \\
\text { принимающих ОК, по сравне- } \\
\text { нию с непринимающим, \% }\end{array}$ \\
\hline Дисменорея & 63 \\
\hline Меноррагия & 48 \\
\hline $\begin{array}{l}\text { Фиброзно-кистозные заболе- } \\
\text { вания молочных желез }\end{array}$ & 50 \\
\hline Рак яичников & $40 / 80^{1}$ \\
\hline $\begin{array}{l}\text { Функциональные кисты яич- } \\
\text { ников }\end{array}$ & $48 / 76^{2}$ \\
\hline Рак матки & 50 \\
\hline Эндометриоз & 50 \\
\hline $\begin{array}{l}\text { Воспалительные заболевания } \\
\text { органов малого таза }\end{array}$ & 60 \\
\hline Ревматоидный артрит & 78 \\
\hline Железодефицитная анемия & 45 \\
\hline $\begin{array}{l}\text { Язвенная болезнь двенадцати- } \\
\text { перстной кишки }\end{array}$ & 40 \\
\hline Рак кишечника & 37 \\
\hline Акне & 25 \\
\hline
\end{tabular}

Примечания: ${ }^{1}$ - риск рака яичников снижается на $40 \%$ nри приеме ОК от 3 до 6 мес. и возрастает до 80 \% при приеме более 10 лет; $^{2}$ - снижение риска выражено в больщей степени при приеме высокодозированных ОК. 
возрастных групп, а также развития инволюционного остеопороза (постменопаузального и сенильного) и его осложнений является формирование оптимального пика костной массы - максимального количество костной ткани, которое набирает человек в течение первых двух десятилетий жизни. По данным некоторых авторов (Cromer B., 2000), 50 \% костной массы накапливается в период препубертата, 35 \% - в пубертатном и постпубертатном периодах, а остальные $15 \%$ - в молодом возрасте. Формирование пика костной массы завершается к 22-24 годам.

В настоящее время существуют противоречивые сведения о влиянии ГК на формирование пика костной массы. Хотя рандомизированных контролированных исследований по изучению влияния ГК на состояние костной ткани у подростков и молодых девушек в существующей литературе не обнаружено, в нескольких проспективных исследованиях показано, что прием ГК достоверно не влияет на формирование пика костной массы. Так, в исследовании, проведенном Lloyd T. et al. (2000), проанализировано влияние приема (в течение $\geq 6$ мес. и продолжающийся до 22 лет) низкодозированных монофазных ОК у 80 пациенток в возрасте от 12 до 20 лет с помощью двухэнергетической рентгеновской денситометрии (ДРА). Результаты данного исследования не выявили достоверных отличий показателей минеральной плотности костной ткани (МПКТ) девушек, получающих ОК, и группы контроля, что свидетельствует об отсутствии какого-либо влияния ОК на формирование пика костной массы.

В нескольких проспективных исследованиях оценена сравнительная эффективность различных режимов ГК у девушек. Так, в 24-месячном исследовании Cromer B.A. et al. (1996) обследовано 48 пациенток в возрасте 12-21 года. Было выделено 4 группы: девушки, получающие ОК, содержащий $30 \mu$ ЭЭ и $150 \mu$ дезогестрела, подкожный имплантат норплант, инъекционный контрацептив депо-провера, и не принимающие ГК (контроль). Через 12 мес. исследования достоверных различий между динамикой МПКТ в группе, принимающей ОK, и контрольной группе не выявлено (показатели МПКТ поясничного отдела позвоночника увеличились соответственно на 1,5\% в 1-й группе и на 2,9\% в контроле). Через год наблюдения в группе пациенток, получающих депо-провера, МПКТ снизилась на 1,5\% по сравнению с увеличением показателей на 2,5 \% в группе, получавшей норплант, и на 2,9 \% в контроле ( $<<0,02)$. После 2 лет наблюдения МПКТ увеличилась на 9,3\% в группе, получавшей норплант, и на 9,5\% в контроле, однако отмечено ее уменьшение на 3,1 \% в группе, получавшей депо-провера ( $<<0,0001)$. Авторами сделан вывод, что депо-провера способствует задержке минерализации костной ткани у подростков, тогда как норплант и комбинированные ОК не влияют на динамику показателей МПКТ.

В другом 24-месячном проспективном исследовании (Lara-Torre E. et al., 2004) обследовано 148 девушек в возрасте 11-21 года, разделенных на следующие группы: принимающие ОК, ДМПА и не принимающие ГК (контроль). Как и в предыдущем исследовании, авторами не выявлено достоверных отличий между показателями МПКТ поясничного отдела позвоночника у пациенток, принимающих ОК, и контролем. Однако за 24 мес. наблюдения в группе, начавшей прием ОK, не установлено достоверных изменений МПКТ по сравнению с исходными данными. У девушек, получавших ДМПА, выявлено статистически достоверное снижение показателей МПКТ по сравнению с контролем через 6 мес. (динамика показателя: $-3,02 \%, \mathrm{p}=0,014$ ), 12 мес. $(-3,38 \%, p=0,001), 18$ мес. $(-4,81 \%, p<0,001)$ и 24 мес. $(-6,81 \%, \mathrm{p}=0,010)$, что свидетельствует о его отрицательной роли в формировании пика костной массы.

Однако в литературе описаны исследования, которые ставят под сомнение отсутствие отрицательного эффекта ГК на формирование пика костной массы. Так, в 12-месячном проспективном исследовании Rome E. et al. (2004) с использованием ДРА и определением биохимических маркеров костного ремоделирования обследовано 370 девушек в возрасте 1218 лет: 165 пациенток принимали ОК, содержащий $20 \mu$ ЭЭ и 100 нг левоноргестрела, 53 получали ДМПА, а 152 девушки, не принимавшие ГК, составили группу контроля. Авторами не выявлено достоверных различий показателей МПКТ поясничного отдела позвоночника и бедренной кости между выделенными группами. При анализе биохимических маркеров ремоделирования костной ткани отмечено достоверное увеличение активности костноспецифической щелочной фосфатазы (ЩФ) в сыворотке крови контрольной группы по сравнению с показателями девушек, принимающих ГК: в контрольной группе показатели $\mathrm{M} \pm \mathrm{m}$ составили соответственно 40,40 \pm 1,03 МЕ/л; в группе, получающей ДМПА, $-35,20 \pm 1,05$ МЕ/л; в группе, принимающей ОK, $-35,50 \pm 1,03$ МЕ/л. Кроме того, установлена тенденция к увеличению $(\mathrm{p}=0,08)$ уровня дезоксипиридинолина (Дпир.) в моче у девушек контрольной группы $(9,9 \pm 1,0$ нмоль/моль креатинина) по сравнению с группой, получающей ДМПА $(9,1 \pm 1,1)$ или ОК $(8,9 \pm 1,3)$, хотя авторами и не выявлена достоверная связь между биохимическими маркерами костного ремоделирования и показателями МПКТ поясничного отдела позвоночника или бедренной кости. Таким образом, увеличение активности маркеров формирования и резорбции костной ткани у девушек, не принимающих ГК, по сравнению с пациентками, принимающими ДМПА или ОК, свидетельствует об угнетении уровня костного метаболизма при приеме данных гормональных контрацептивов.

В другом 12-месячном проспективном сравнительном исследовании, проведенном Cromer B.A. et al. (2004), с участием 215 девушек в возрасте 12-18 лет, которые были разделены на 3 группы в зависимости от вида ГК (получающие ОК, содержащий $20 \mu$ ЭЭ и 100 нг левоноргестрела, ДМПА и не принимающие ОК (контроль)), установлено достоверное увеличение показателей МПКТ поясничного отдела позвоночника и шейки бедренной кости по данным ДРА в группе, принимавшей ОК, и в контроле, однако динамика пока- 
зателей в группе, принимавшей ОК, была достоверно меньшей по сравнению с показателями контрольной группы. Через 12 мес. наблюдения МПКТ в поясничном отделе позвоночника снижалась в группе, получавшей ДМПА (динамика показателя: $-1,4 \%$; $95 \%$ ДИ $-2,73 ;-0,10)$, и увеличивалась на 3,8 \% (95\% ДИ 3,11 ; $4,57)$ в контрольной группе ( $<<0,001)$. Увеличение показателей МПКТ поясничного отдела позвоночника в группе, принимавшей ОК, составило 2,3 \% (95\% ДИ $1,49 ; 3,18)$ и было достоверно меньшим по сравнению с показателями контрольной группы $(\mathrm{p}=0,03)$. Динамика показателей МПКТ шейки бедренной кости составила соответственно -2,2 \% (95\% ДИ -3,95; -0,39) в группе, принимающей ДМПА, 0,3 \% (95\% ДИ -0,87; $1,41)$ в группе, принимающей ОК, а средние показатели были достоверно ниже (соответственно $\mathrm{p}<0,001$ и $\mathrm{p}=0,03)$ по сравнению с показателями контрольной группы (2,3\%; $95 \%$ ДИ 1,29; 3,27).

В 60-месячном проспективном исследовании (Polatti F. et al., 1995) изучено влияние ОК, содержащего $20 \mu$ ЭЭ и 0,15 мг дезогестрела, на формирование пика костной массы у 200 молодых женщин в возрасте 19-22 лет. Авторами установлено отсутствие динамики МПКТ поясничного отдела позвоночника в группе, получающей ОK, и ее увеличение в контрольной группе. Выявлено также отсутствие динамики активности костноспецифической кислой фосфатазы сыворотки крови и соотношения уровня гидроксипролина и креатинина мочи в обеих группах. Через 5 лет приема ЭЭ и дезогестрела не обнаружено достоверных изменений показателей МПКТ в первой группе, тогда как в контроле установлено достоверное увеличение показателей МПКТ $(+7,8 \%, \mathrm{p}<0,01)$ на фоне отсутствия достоверных отличий уровней биохимических маркеров ремоделирования костной ткани по сравнению с исходными показателями в обеих группах, что дало авторам основание говорить о наличии отрицательного влияния приема данного ОК на формирование пика костной массы у молодых женщин.

Таким образом, существующие в настоящее время в литературе неоднозначные данные свидетельствуют о необходимости проведения рандомизированных исследований по изучению влияния ГК различных групп на формирование пика костной массы.

\section{Эффект гормональной контрацепции на состояние костной ткани здоровых женщин репродуктивного возраста}

В настоящее время существуют противоречивые данные относительно влияния ГК на показатели МПКТ здоровых женщин. Многочисленные исследования как подтверждают его (Lindsay R. et al., 1986; Kleerekoper M. et al., 1991; Laitinen K. et al., 1991; Recker R.R. et al., 1992; Pasco J.A. et al., 2000; Berenson A.B. etal., 2001; Cobb K.L. etal., 2002; Wallace L.S., Ballard J.E., 2002; Elgán C. et al., 2003; Ruffing J.A., Nieves J.W., Zion M. et al., 2007; Berenson A.B., Rahman M., Breitkopf C.R., 2008; Allali F., El Mansouri L., Abourazzak F.-Z. et al., 2009; Scholes D., Ichikawa L., La Croix A.Z. et al., 2010; Bedford J.L., Barr S.I., 2010; Scholes D., Hubbard R.A.,
Ichikawa L.E. et al., 2011), так и отрицают (Polatti F. et al., 1995; Hartard M. et al., 1997; Burr D.B. et al., 2000; Weaver C.M. et al., 2001; Prior J.C. et al., 2001; Cromer B.A. et al., 2004; Hartard M. et al., 2004; Monteiro-Dantas C., Espejo-Arce X., Lui-Fil J.F. et al., 2007; Cromer B.A., Bonny A.E., Stager M. et al., 2008). Также в литературе существует много данных, свидетельствующих об отсутствии какого-либо эффекта гормональных контрацептивов на показатели МПКТ у женщин репродуктивного возраста с нормальными показателями костной ткани (Lloyd T. et al., 2000; Petitti D.B. et al., 2000; Ott S.M. et al., 2001; Perrotti M. et al., 2001; Hawker G.A. et al., 2002; Wanichsetakul P. et al., 2002; Reed S.D. et al., 2003; LaraTorre E. et al., 2004; Lloyd T. et al., 2004; Berenson A.B. et al., 2004; Paoletti A.M. et al., 2004; Rome E. et al., 2004; Afghani A. et al., 2004; Meyer N.L. et al., 2004).

Так, в одномоментном эпидемиологическом исследовании, проведенном еще в середине 70-х годов прошлого века (Goldsmith N.F., Johnston J.O., 1975), с помощью ${ }^{125}$ І фотонной абсорбциометрии дистального отдела лучевой кости изучен эффект приема ОК у 2199 женщин в возрасте 15-79 лет. Исследователями было выделено 4 группы: женщины, принимающие ОК, содержащие $\geq 100$ или < $100 \mu$ гестранола, 50-100 нг ЭЭ и не принимающие ОК. Показано, что прием ОК, содержащих $\geq 100 \mu$ местранола, увеличивает костную минерализацию, однако подобного эффекта не выявлено при приеме ОК, содержащих 50-80 нг местранола или 50-100 $\mu$ г ЭЭ.

В последующих исследованиях было подтверждено положительное действие ОК на структурно-функциональное состояние костной ткани. Показано, что МПКТ поясничного отдела позвоночника у молодых здоровых женщин, принимающих ОК, увеличивается ежегодно приблизительно на $1 \%$ (Lindsay R. et al., 1986). В одномоментном исследовании, проведенном Lindsay R. et al. (1986) с участием 57 женщин в возрасте 25-35 лет, принимавших когда-либо ОК (30 или $50 \mu$ ЭЭ в сочетании с норгестрелом) или никогда их не принимавших, показано, что показатели МПКТ поясничного отдела позвоночника у женщин первой группы были на 12 \% выше по сравнению с пациентками, не принимающими ОК.

Еще одно подобное исследование проведено Pasco J.A. et al. (2000) с участием 710 австралийских женщин в возрасте 20-69 лет. Для анализа было выделено 2 группы: принимавшие когда-либо ОК ( $=579)$ и никогда не принимавшие ОК $(n=131)$. В результате данного исследования показано, что МПКТ поясничного отдела позвоночника у пременопаузальных женщин, принимавших когда-либо ОК, была на 3,3 \% выше $(\mathrm{p}=0,014)$ по сравнению с показателями пациенток, никогда не принимавших ОK, хотя подобные отличия у постменопаузальных женщин не были выявлены. Более высокие показатели МПКТ ассоциированы с возрастанием длительности приема ОК (с увеличением в среднем на 3,2 \% за 5 лет приема и с последующим увеличением на $0,2 \%$ при приеме $\geq 5$ лет). В других измеряемых областях подобная ассоциация не выявлена. 
При изучении связи между приемом ОК и показателями МПКТ в ретроспективном исследовании Kleerekoper M. et al. (1991) с использованием двух методов определения состояния костной ткани - однофотонной абсорбциометрии лучевой кости и ДРА поясничного отдела позвоночника - при обследовании 2297 женщин, из которых 29,7 \% принимали когда-либо ОК, выявлена достоверная связь между длительностью приема ОК и показателями МПКТ (наиболее выраженной эта связь была у женщин, принимающих $\mathrm{OK} \geq 10$ лет). Это дало авторам основание утверждать, что прием ОК является защитным фактором, предупреждающим снижение показателей МПКТ (относительный риск (ОР) снижения МПКТ составил 0,35, 95\% ДИ 0,23; 0,53). Подобные данные были получены и при обследовании 293 финских женщин в возрасте 20-76 лет (Laitinen K. et al., 1991). В группе пременопаузальных женщин выявлена достоверная корреляционная связь между приемом ОК и показателями МПКТ. В одномоментном исследовании Wallace L.S., Ballard J.E. (2002) также выявлена достоверная корреляционная связь между приемом ОК и МПКТ трохантера и всей бедренной кости.

В одномоментном исследовании Cobb K.L. et al. (2002) с участием 476 женщин негроидной и европеоидной расы в возрасте 18-30 лет выявлена достоверная корреляционная связь между МПКТ позвоночника и кумулятивной дозой ОК у женщин европеоидной расы, но не у пациенток негроидной расы. Кумулятивная доза эстрогенов, содержащихся в ОК, была связана с увеличением МПКТ позвоночника (на 4,0 \%, $\mathrm{p}=0,024)$ у женщин европеоидной расы, но не у женщин негроидной расы и ассоциирована со снижением риска низкой МПКТ (нижний квартиль) в позвоночнике, бедренной кости и всем скелете у женщин европеоидной расы. При сравнении показателей МПКТ женщин в зависимости от кумулятивной дозы ОК (самый высокий и самый низкий квартиль) показатель ОР составил для поясничного отдела позвоночника 0,08 (95\% ДИ 0,02; 0,46), бедренной кости - 0,23 (0,06; $0,87)$; всего скелета - 0,37 $(0,11 ; 1,26)$, однако прием OK не был связан с низкими показателями МПКТ у женщин негроидной расы.

Несмотря на вышеизложенное, в настоящее время некоторые одномоментные исследования, существующие в литературе, не подтверждают положительный эффект гормональных контрацептивов на показатели МПКТ у молодых здоровых женщин. Так, в одномоментном исследовании Lloyd T. et al. (2000, 2004), проведенном с использованием количественной компьютерной томографии поясничного отдела позвоночника у 25 женщин, которые были разделены на 2 группы: принимающие ОК ( $\geq 50 \mu г / д$ местранола) в течение $\geq 67$ мес. и не принимающие ОК, достоверных различий показателей МПКТ в зависимости от приема ОК не выявлено (соответственно $160,6 \pm 6,9$ в контроле и 161,2 \pm 7,4 мг/мл у женщин, принимающих ОK, $\mathrm{p}=0,87)$.

В одномоментном исследовании (Hawker G.A. et al., 2002) с участием 830 женщин, принимавших участие в Nord-Trøndelag Health Study (Норвегия, 1995-1997) в возрасте 19-35 лет, разделенных на 3 группы: пациентки, принимающие ОК в настоящем, принимавшие OK в прошлом и никогда не принимавшие OK, с использованием однофотонной рентгеновской абсорбциометрии показано, что МПКТ ультрадистального отдела недоминантной лучевой кости достоверно не отличалась в группах и составила соответственно 0,386 г $/ \mathrm{cm}^{2}$ (95\% ДИ 0,380, 0,391) в первой, 0,385 (95\% ДИ $0,381,0,389)$ - во второй и 0,388 (95\% ДИ 0,378, $0,400)$ - в третьей группе.

Подобные результаты подтверждаются и в других одномоментных исследованиях: Sowers M. et al. (1985); Hreschyshyn M.M. et al. (1988); Hall M.L. et al. (1990); Petitti D.B. et al. (2000); Perotti M. et al. (2001); Wanichsetakul P. et al. (2002), в которых использовали различные методы исследования состояния костной ткани - 125 I фотонная абсорбциометрия, однофотонная рентгеновская абсорбциометрия лучевой кости и двухэнергетическая рентгеновская денситометрия поясничного отдела позвоночника и шейки бедренной кости. Отсутствие связи между показателями МПКТ и приемом ОК подтверждается и в некоторых эпидемиологических исследованиях (Stevenson J.C. et al., 1989; Murphy S. et al., 1993; Hawker G.A. et al., 2002; Afghani A. et al., 2004; Meyer N.L. et al., 2004). Однако в части из них на фоне отсутствия достоверных различий показателей состояния костной ткани выявляются различия в уровне биохимических маркеров костного ремоделирования в зависимости от приема ГК. Так, в одномоментном исследовании, проведенном Garnero P. et al. (1995), с участием 208 женщин в возрасте 35-49 лет достоверных отличий показателей МПКТ у пациенток, принимающих ГК когда-либо и никогда не принимавших ГК, не выявлено. Однако установлено снижение уровня маркеров костной резорбции и формирования во всех группах, принимающих контрацептивы, по сравнению с контролем: остеокальцин $-7,7 \pm 2,7$ и $10,1 \pm 3,1$ нг/мл $(-24 \%, \mathrm{p}<0,001)$; костноспецифическая ЩФ - 7,5 $\pm 2,3$ и $8,8 \pm 2,7$ нг/мл $(-15 \%$, p < 0,003); С-терминальный пропептид коллагена I типа $-77,2 \pm 93,1$ и 93,1 $\pm 31,9$ нг/мл $(-17 \%, \mathrm{p}=0,001)$, пиридинолин - $175 \pm 91$ и $211 \pm 105$ мкг/ммоль креатинина $(-17 \%, \mathrm{p}=0,03)$ и $\mathrm{N}$-телопептид $-16,2 \pm 5,9$ и $22,5 \pm$ 9,4 нмоль эквивалент костного коллагена/моль креатинина $(-28 \%, \mathrm{p}<0,001)$.

В другом одномоментном исследовании (Ott S.M. et al., 2001) изучено влияние ГК на маркеры ремоделирования костной ткани в зависимости от режима гормональной контрацепции. Авторами обследовано 227 женщин в возрасте 18-39 лет с разделением на группы: женщины, принимающие ОК (53,6 \%, 35 нг ЭЭ и 0,5-1 мг норэтидрона; $18 \%$, 35 г ЭЭ и 1 мг левоноргестрела или 1 мг эстрадиола диацетата; 13,7 \%, $30 \mu$ ЭЭ и 1,5 мг норэтидрона; 9,7 \%, 20 нг ЭЭ и левоноргестрела или норэтидрона), принимающие ДМПА или не принимающие ГК (контроль). На фоне отсутствия достоверных различий показателей МПКТ поясничного отдела позвоночника $\left(1,086 \pm 0,085\right.$ г $/ \mathrm{cm}^{2}$ в груп-

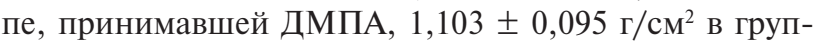

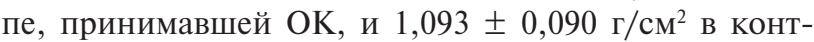


роле, $\mathrm{p}=0,051)$ и всего скелета между группами установлено снижение уровня остеокальцина и N-телопептида у женщин, принимающих ОК, по сравнению с контролем, подтверждающее снижение интенсивности костной резорбции. Уровень $\mathrm{N}$-телопептида составил 42,4 $\pm 2,3$ нмоль/ммоль креатинина в группе, принимающей ДМПА, 26,2 \pm 3,3 нмоль/моль в группе, принимающей ОK, и 35,4 $\pm 2,9$ нмоль/моль в контроле (отличия по сравнению с контролем были достоверны во всех группах). Уровень остеокальцина также снижался, однако различия в группе, принимавшей ДМПА, и контроле были недостоверными, а уровень паратиреоидного гормона не различался в группах в зависимости от приема ГК.

Ни одно из обнаруженных в литературе 4 рандомизированных исследований по влиянию ГК на состояние КТ у женщин репродуктивного возраста (табл. 4) не подтвердило положительный эффект ГК на показатели МПКТ, измеренной с помощью двухэнергетической рентгеновской денситометрии (Castelo-Branco C. et al., 1998; Nappi C. et al., 2003; Nappi C. et al., 2005) или компьютерной томографии (Endrikat J. et al., 2004). Однако в трех из них установлен положительный эффект ОК на показатели костного метаболизма, в частности уровни маркеров костной резорбции (пиридинолина (Пир) и деоксипиридинололина (ДПир)) (Nappi C. et al., 2003; Nappi C. et al., 2005) и N-телопептида (Endrikat J. et al., 2004). Рандомизированных исследований, подтверждающих отрицательный эффект ГК на состояние костной ткани у молодых здоровых женщин, в литературных источниках не выявлено. В проспективных исследованиях, изучающих влияние различных режимов ГК на состояние костной ткани у молодых здоровых женщин, также получены неоднозначные результаты (табл. 4).

При изучении биохимических маркеров ремоделирования костной ткани в проспективных исследованиях также продемонстрирован положительный эффект ОК. Так, в 12-месячном проспективном исследовании с участием 30 женщин в возрасте 22-30 лет (Paoletti A.M. et al., 2000), разделенных на 3 группы: принимающие $20 \mu$ ЭЭ $+75 \mu \mathrm{g}$ гестодена, $30 \mu$ г ЭЭ + 75 нг гестодена и контроль, установлено снижение уровня Пир., Дпир. в группе, принимающей ОK, свидетельствующее о снижении темпов костной резорбции.

В другом проспективном исследовании (Kitai E. et al., 1992) биохимические маркеры костного метаболизма были проанализированы у 30 женщин (средний возраст - 23,7 года) в зависимости от факта приема ОК. Установлено снижение уровня экскреции кальция по отношению к креатинину мочи в группе, принимающей ОК, что свидетельствует, по мнению авторов, о снижении темпов костной резорбции.

В литературе описан ряд исследований, свидетельствующих об отрицательном влиянии ОК на состояние костной ткани у молодых здоровых женщин. В популяционном мультицентровом одномоментном исследовании Canadian Multicentre Osteoporosis Study (Prior J.C. et al., 2001) обследованы 524 канадские женщи- ны в возрасте 25-45 лет (средний возраст - 36,3 года), из которых 454 пациентки принимали ОК (средний возраст начала приема ОК - 19,8 года, средняя длительность приема $-6,8$ года). Для анализа были выделены слудующие группы: женщины, принимающие когда-либо ОK ( $\geq 3$ мес.), никогда не принимавшие OK (или от 0 до $<3$ мес.). В результате данного исследования установлено, что показатели МПКТ поясничного отдела позвоночника и трохантера у женщин, принимавших когда-либо ОК, по сравнению с пациентками, никогда их не принимавшими, были достоверно ниже. МПКТ (M $\pm \mathrm{SD})$ поясничного отдела позвоночника у женщин, принимавших ОК, составила

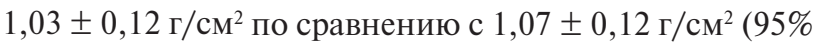
ДИ $-0,07 ;-0,001)$ у пациенток, никогда не принимавших ОК, при этом показатели МПКТ женщин, принимающих ОК в настоящем или в прошлом, не отличались между собой. Авторами не выявлено связи между МПКТ и длительностью приема ОК, а также возрастом начала их приема, что ставит под сомнение возможность положительного действия ОК на состояние костной ткани.

Интересными представляются исследования, изучающие влияние ОК на состояние костной ткани молодых здоровых женщин в зависимости от уровня их физической активности. Так, в одномоментном исследовании Hartard M. et al. (1997) обследованы молодые женщины в возрасте 20-35 лет, разделенные на 4 группы: длительно занимающиеся физическими упражнениями и краткосрочно принимающие ОК; длительно занимающиеся физупражнениями и долгосрочно принимающие ОK; занимающиеся физупражнениями краткосрочно и длительно принимающие ОК и краткосрочно занимающиеся физупражнениями и краткосрочно принимающие ОК. Установлено, что наиболее высокими показатели МПКТ были в группе женщин, длительно занимающихся физупражнениями $(9,5 \pm 4,3$ года) и краткосрочно принимающих ОK $(1,6 \pm 1,7$ года). Однако в данном исследовании авторами не выявлено положительного эффекта умеренных физнагрузок у женщин, длительно занимающихся физупражнениями $(10,40 \pm 4,14$ года) и длительно принимающих ОK $(8,20 \pm 4,14$ года). Не обнаружено также достоверных различий показателей во всех исследуемых регионах скелета в группе занимающихся краткосрочно физупражениями и длительно или краткосрочно принимающих ОК, в результате чего авторами сделан вывод, что прием ОК отрицательно влияет на положительный эффект занятий физическими упражнениями.

В ретроспективном исследовании Hartard M. et al. (2004) проанализировано состояние костной ткани у 69 женщин-атлетов в возрасте 18-35 лет, занимающихся различными видами спорта, тренирующими выносливость организма. Анализ проводили в 2 группах: женщины, принимавшие ОК (прием > 3 лет у женщин в возрасте $<22$ лет или прием $>50 \%$ времени от менархе в возрасте 22-35 лет), и пациентки, не принимавшие ОК (контроль). В результате данного исследования установлено, что показатели МПКТ у женщин, при- 
Таблица 4. Проспективные исследования, изучающие эффект гормональных контрацептивов на показатели костной ткани у молодых здоровых женщин

\begin{tabular}{|c|c|c|}
\hline Авторы & Дизайн исследования & Результаты \\
\hline $\begin{array}{l}\text { Castelo-Branco C. } \\
\text { et al., } 1998\end{array}$ & $\begin{array}{l}\text { 24-мес. рандомизированное контролируемое исследова- } \\
\text { ние. } 67 \text { женщин в возрасте } 19-29 \text { лет. } 2 \text { группы: } 35 \mu г \text { ЭЭ } \\
+2 \text { нг ципротерона ацетата }(n=35), 30 \mu г \text { ЭЭ + } 150 \mu г \text { де- } \\
\text { зогестрела }(\mathrm{n}=32) . \text { МИ: ДРА }\end{array}$ & $\begin{array}{l}\text { Не выявлено достоверных изменений МПКТ по сравне- } \\
\text { нию с исходными данными в какой-либо из групп через } \\
12 \text { и } 24 \text { мес. наблюдения }\end{array}$ \\
\hline $\begin{array}{l}\text { Nappi C. et al., } \\
2003\end{array}$ & $\begin{array}{l}\text { 12-мес. рандомизированное контролируемое исследо- } \\
\text { вание. } 56 \text { женщин в возрасте } 22-34 \text { лет. } 3 \text { группы: } 20 \mu г \\
\text { ЭЭ }+75 \mu \text { гестодена }(n=19), 15 \mu г \text { ЭЭ }+60 \text { нг гестоде-- } \\
\text { на }(n=18), \text { контроль }(n=19) \text {. МИ: ДРА (поясничный от- } \\
\text { дел позвоночника), БХМ (Пир., Дпир. (моча), остеокальцин } \\
\text { (кровь)) }\end{array}$ & $\begin{array}{l}\text { Не выявлено достоверных изменений МПКТ по сравне- } \\
\text { нию с исходными данными в какой-либо из групп. Отмече- } \\
\text { но достоверное уменьшение уровня Пир., Дпир. в группах, } \\
\text { получавших ОК, через } 6,9 \text { и } 12 \text { мес. по сравнению с ис- } \\
\text { ходными показателями }(p<0,05)\end{array}$ \\
\hline $\begin{array}{l}\text { Endrikat J. et al., } \\
2004\end{array}$ & 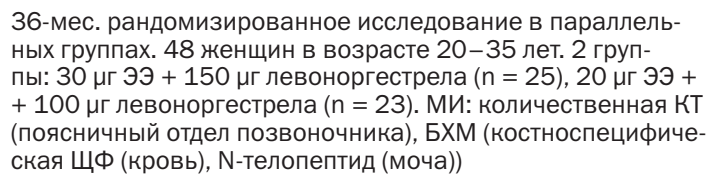 & $\begin{array}{l}\text { Не выявлено достоверных изменений МПКТ по срав- } \\
\text { нению с исходными данными в обеих группах. Отмече- } \\
\text { но уменьшение уровня N-телопептида в обеих группах, } \\
\text { что свидетельствует о снижении темпов резорбции кост- } \\
\text { ной ткани }\end{array}$ \\
\hline $\begin{array}{l}\text { Nappi C. et al., } \\
2005\end{array}$ & $\begin{array}{l}\text { 12-мес. рандомизированное контролируемое исследова- } \\
\text { ние. } 71 \text { женщина в возрасте } 22-34 \text { лет. } 3 \text { группы: } 30 \text { нг ЭЭ } \\
+3 \text { мг дроспиренона }(n=24), 30 \mu г \text { ЭЭ + } 75 \text { нг гестодена } \\
\text { (n = 24), контроль }(n=23) \text { МИ: ДРА (поясничный отдел по- } \\
\text { звоночника), БХМ }\left(\mathrm{Ca}^{2+} \text {, остеокальцин (кровь), Са }{ }^{2+} \text {, Пир., }\right. \\
\text { Дпир. (моча)) }\end{array}$ & $\begin{array}{l}\text { Отмечено достоверное снижение уровня Пир. и Дпир. в } \\
\text { обеих группах, принимавших ОК, через 6, } 9 \text { и } 12 \text { мес. по } \\
\text { сравнению с исходными показателями, свидетельствую- } \\
\text { щее о снижении темпов резорбции КТ, тенденция к уве- } \\
\text { личению показателей МПКТ в группе, принимающей ЭЭ + } \\
\text { дроспиренон }\end{array}$ \\
\hline $\begin{array}{l}\text { Recker R.R. et al., } \\
1992\end{array}$ & $\begin{array}{l}\text { 5-летнее проспективное исследование } 156 \text { женщин - уча- } \\
\text { щихся колледжа. } 3 \text { группы: принимающие ОК в настоящем } \\
(\mathrm{n}=34), \text { прошлом }(n=43) \text { и никогда не принимавшие ОК. } \\
\text { МИ: ОФА (лучевая кость), ДФА (позвоночник, весь скелет) }\end{array}$ & $\begin{array}{l}\text { МПКТ всего скелета (но не МПКТ позвоночника и луче- } \\
\text { вой кости) положительно коррелировала с приемом ОК } \\
(r=0,31, p=0,01)\end{array}$ \\
\hline $\begin{array}{l}\text { Berenson A.B. } \\
\text { et al., } 2001\end{array}$ & $\begin{array}{l}\text { 12-мес. проспективное исследование. } 155 \text { бело- и темноко- } \\
\text { жих женщин-военнослужащих в возрасте } 18 \text { - } 33 \text { лет. } 4 \text { груп- } \\
\text { пы: } 35 \text { нг ЭЭ + } 1 \text { мг норэтидрона }(n=28), 30 \mu г \text { ЭЭ +0,15 мг } \\
\text { дезогестрела (n = 35), } 150 \text { мг ДМПА ( }=33), \text { контроль } \\
\text { (n = 59). МИ: ДРА (поясничный отдел позвоночника) }\end{array}$ & 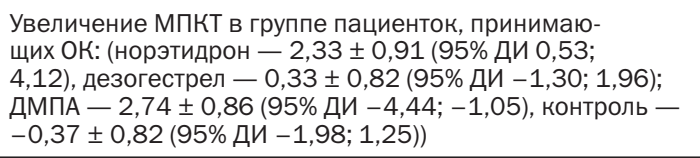 \\
\hline $\begin{array}{l}\text { Elgán C. et al., } \\
2003\end{array}$ & $\begin{array}{l}\text { 24-мес. проспективное исследование. } 118 \text { женщин в воз- } \\
\text { расте 18-26 лет. } 4 \text { группы: некурящие и не принимающие } \\
\text { ОК (n = 35), курящие и не принимающие ОК ( } n=9) \text {, не куря- } \\
\text { щие и принимающие ОК ( }(=57) \text {, курящие и принимающие } \\
\text { ОК (n= 17). МИ: ДРА (пяточная кость); БХМ: Дпир. (моча) }\end{array}$ & $\begin{array}{l}\text { Прием ОК нивелирует отрицательное влияние курения на } \\
\text { МПКТ. Исходные и конечные показатели МПКТ у женщин, } \\
\text { принимающих ОК, были более высокими по сравнению с } \\
\text { пациентками, не принимающими их. Курение ассоциро- } \\
\text { вано с выраженным отрицательным влиянием на МПКТ. } \\
\text { Показатели МПКТ у женщин с нормальным менструаль- } \\
\text { ным циклом, использующих ОК, были достоверно выше до } \\
\text { лечения и по его завершении. МПКТ у женщин с нерегу- } \\
\text { лярным менструальным циклом, принимающих ОК, улуч- } \\
\text { шалась под влиянием приема ОК. Уровень Дпир. отличал- } \\
\text { ся в группах и был ассоциирован с приемом ОК }\end{array}$ \\
\hline $\begin{array}{l}\text { Mazess R.B., } \\
\text { Barden H.S., } \\
1991\end{array}$ & $\begin{array}{l}\text { 24-мес. проспективное исследование. } 300 \text { женщин в воз- } \\
\text { расте } 20-39 \text { лет. } 50 \% \text { женщин, принимающих ОК в прош- } \\
\text { лом или настоящем, } 50 \text { \% женщин, никогда не принимав- } \\
\text { ших ОК. МИ: ДРА (поясничный отдел позвоночника), ОФА } \\
\text { (лучевая кость) }\end{array}$ & $\begin{array}{l}\text { Связь между показателями МПКТ и приемом ОК не вы- } \\
\text { явлена }\end{array}$ \\
\hline $\begin{array}{l}\text { Reed S.D. et al., } \\
2003\end{array}$ & $\begin{array}{l}\text { 36-мес. проспективное исследование. } 245 \text { женщин в } \\
\text { возрасте } 18-39 \text { лет. } 2 \text { группы: женщины, принимающие } \\
\text { ОК в настоящем (80\%, 30-35 нг ЭЭ) (n= 89), контроль } \\
\text { (n = 156). МИ: ДРА (поясничный отдел позвоночника, прок- } \\
\text { симальный отдел бедренной кости, весь скелет) }\end{array}$ & $\begin{array}{l}\text { Изменения МПКТ по сравнению с исходными показателя- } \\
\text { ми не выявлены }\end{array}$ \\
\hline $\begin{array}{l}\text { Berenson A.B. } \\
\text { et al., } 2004\end{array}$ & $\begin{array}{l}\text { 24-мес. проспективное исследование. } 191 \text { женщина в воз- } \\
\text { расте } 18-33 \text { лет. Принимающие ОК (35 } \mu г \text { ЭЭ }+1 \text { мг норэ- } \\
\text { тидрона или } 30 \mu г \text { ЭЭ + 0,15 мг дезогестрела }(n=86)) \text {, } \\
\text { ДМПА }(n=47), \text { контроль }(n=58) \text {. МИ: ДРА (поясничный от- } \\
\text { дел позвоночника) }\end{array}$ & $\begin{array}{l}\text { Достоверных отличий МПКТ между пациентками, получа- } \\
\text { ющими ОК, и контролем не выявлено. У женщин, прини- } \\
\text { мающих дезогестрел, отмечено снижение МПКТ на 2,6 \% } \\
\text { за } 24 \text { мес. по сравнению с исходными данными. Измене- } \\
\text { ния МПКТ в группе, получавшей норэтидрон, были недо- } \\
\text { стоверными по сравнению с контролем }\end{array}$ \\
\hline $\begin{array}{l}\text { Paoletti A.M. } \\
\text { et al., } 2004\end{array}$ & $\begin{array}{l}\text { 6-мес. проспективное исследование. } 54 \text { женщины в воз- } \\
\text { расте } 20-30 \text { лет. } 2 \text { группы: } 30 \text { нг ЭЭ + } 3 \text { мг дроспиренона } \\
\text { (n= 28), контроль ( } \mathrm{n}=26) \text {. МИ: ДРА (пяточная кость), БХМ } \\
\text { (остеокальцин, костноспецифическая ЩФ (кровь), Пир., } \\
\text { Дпир. (моча)) }\end{array}$ & $\begin{array}{l}\text { Достоверных изменений показателей МПКТ по сравне- } \\
\text { нию с исходными данными в обеих группах не выявлено. } \\
\text { В отличие от показателей контроля в группе, получающей } \\
\text { ОК, установлено достоверное уменьшение уровня остео- } \\
\text { кальцина, костноспецифической щФ, Пир., Дпир. }\end{array}$ \\
\hline
\end{tabular}

Примечания: ЭЭ - этинилэстрадиол; МПКТ - минеральная плотность костной ткани; ОК - оральные контрацептивы; КТ - костная ткань; МИ - методы исследования; ДРА - двухэнергетическая рентгеновская абсорбциометрия; БХМ - биохимические методы; КТ - компьютерная томография; ОФА - однофотонная абсорбциометрия; ДФА - двухфотонная абсорбциометрия; Пир. - пиридинолин, Дпир. - дезоксипиридинолин; щФ - щелочная фосфатаза; $\mathrm{Ca}^{2+}$ - ионизированный кальций. 
нимающих ОК, были на 7,9 \% ниже в поясничном отделе позвоночника и на 8,8 \% в проксимальном отделе бедренной кости по сравнению с контролем (p $<0,01$ для обоих показателей). По данным пошагового регрессионного анализа, авторами было установлено, что раннее начало приема ОК играет отрицательную роль в потере МПКТ у здоровых женщин-атлетов.

В 24-месячном проспективном исследовании Burr D.B. et al. (2000) изучено влияние приема ГК и физических упражнений на состояние костной ткани 123 молодых здоровых женщин в возрасте 18-31 года. Для анализа были выделены следующие группы: не занимающиеся физупражениями и не принимающие ОK; не занимающиеся физупражениями и принимающие $\leq 50 \mu$ ЭЭ; занимающиеся физупражнениями (три занятия в неделю аэробными и анаэробными упражнениями в течение 2 лет) и не принимающие ОK; занимающиеся физупражнениями и принимающие $\leq 50 \mu$ г ЭЭ. В результате данного исследования установлено, что только прием ОК или занятия физическими упражнениями связаны с угнетением нормального увеличения МПКТ шейки бедренной кости у женщин в этом возрастном периоде, однако комбинация ОК и физических упражнений имеет менее выраженный отрицательный эффект на состояние костной ткани. Через 24 мес. наблюдения у женщин, не принимающих ОК и не занимающихся физупражнениями, отмечено наиболее выраженное увеличение МПКТ $(2,07 \pm 2,09 \%)$ и низкий индекс переломов $(8,03 \pm 2,03 \%)$. У пациенток, занимающихся физупражнениями и не принимающих OK, выявлено наиболее выраженное уменьшение всех изучаемых показателей, тогда как показатели женщин, принимающих ОК, независимо от занятий физическими упражнениями были средними.

В другом проспективном исследовании (Weaver C.M. et al., 2001) у 179 женшин в возрасте 18-31 года также оценен эффект сочетанного приема ОК и умеренных физнагрузок на состояние костной ткани. Как и в предыдущем исследовании, женщины были поделены на группы: не занимающиеся физупражениями и не принимающие ОK; не занимающиеся физупражениями, но принимающие $\leq 50$ нг ЭЭ; занимающиеся физупражениями (60 мин/нед - занятия со скакалкой) и принимающие ОK; занимающиеся физупражениями и принимающие ОК ( $\leq 50 \mu$ ЭЭ). В результате данного исследования показано, что положительная динамика показателей МПКТ поясничного отдела позвоночника у женщин, занимающихся физупражнениями, была более выраженной по сравнению с показателями пациенток, не занимающихся физупражнениями, через 6 и 24 мес. наблюдения. У пациенток, принимающих ОК, биохимические показатели ремоделирования костной ткани были ниже в начале исследования и снижались более выраженно по сравнению с женщинами, не принимающими ОК, к 24-му мес. наблюдения. МПКТ поясничного отдела позвоночника снижалась у женщин, занимающихся упражнениями и принимающих ОК, к 6-му мес. и оставалась достоверно более низкой, чем у не занимающихся и принимающих ОK, через 2 года наблюдения.
При сравнении влияния двух различных гестагенсодержащих методов ГК (ДМПА или норплант) на состояние костной ткани у женшин в возрасте 20-45 лет в рандомизированном 6-месячном исследовании (Naessen T. et al., 1995) показано, что МПКТ проксимального отдела предплечья увеличивалась на 2,94 \% $(\mathrm{p}=0,006)$ по сравнению с исходными у пациенток, использующих норплант, тогда как показатели у женщин, принимающих ДМПА, достоверно не изменялись $(-0,41 \%, \mathrm{p}>0,05)$. Через 6 мес. наблюдения различия показателей МПКТ в группах были достоверными и составили для МПКТ проксимального 3,4 \% (95\% ДИ 1,$3 ; 5,5 ; \mathrm{p}=0,03)$ и дистального отдела предплечья 4,1 \% (95\% ДИ 1,3; 9,6; p = 0,08). Изменения МПКТ коррелировали с динамикой биохимических маркеров костного ремоделирования. Так, в группе, принимавшей норплант, установлено увеличение активности и уровней маркеров формирования костной ткани (щелочной фосфатазы и остеокальцина $-1,88-$ 2,26 мккат/л $(\mathrm{p}=0,004)$ и 1,22-3,05 мкг/л $(\mathrm{p}=0,007)$ соответственно). Авторами сделан вывод, что прием левоноргестрела в виде подкожного имплантата норпланта положительно влияет на состояние костной ткани, а прием ДМПА не оказывает какого-либо влияния на состояние костной ткани.

В мультицентровом одномоментном исследовании (Petitti D.B. et al., 2000), включавшем обследование 2474 женщин в возрасте 30-34 лет, среди которых 350 принимали когда-либо ДМПА, 610 - левоноргестрел, а 695 составили контрольную группу, было показано, что МПКТ лучевой кости была достоверно ниже в группе, принимающей краткосрочно ДМПА или левоноргестрел по сравнению с показателями контроля, однако не установлено достоверных различий показателей МПКТ в группах между пациентками, получающими ГК в прошлом (4 года и более) и никогда не получавшими их.

В одномоментном исследовании Wanichsetakul P. et al. (2002), также проведенном на 155 молодых здоровых женщинах в возрасте 30-34 лет, показано, что на фоне отсутствия достоверных различий показателей МПКТ поясничного отдела позвоночника в группе, принимающей ОК, по сравнению с контролем выявлено достоверное ухудшение показателей в группе, принимавшей ДМПА (минимальная длительность приема 24 мес.), $-1,031 \pm 0,090$ по сравнению с $1,096 \pm 0,116$ г $/ \mathrm{cm}^{2}, p=0,007$. Однако во всех других измеряемых местах (шейка бедренной кости, треугольник Варда, трохантер, лучевая и локтевая кость) показатели в группе, получающей ДМПА, не отличались от соответствующих в контроле.

В 24-месячном проспективном исследовании (Berenson A.B. et al., 2001, 2004) у 191 женщины в возрасте 18-33 лет проведена сравнительная оценка влияния различных режимов гормональной контрацепции (35 $\mu$ Г ЭЭ + 1 мг норэтидрона; $30 \mu$ ЭЭ + 0,15 мг дезогестрела, ДМПА) по сравнению с пациентками, не принимающими ОК. Через 12 мес. наблюдения отмечено увеличение МПКТ поясничного отдела позвоночника в группе пациенток, принимающих ОK, и 
ее снижение у пациенток, получающих ДМПА: норэ-

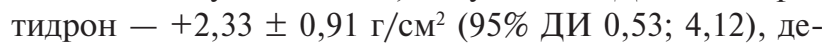

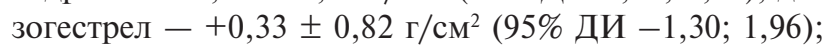

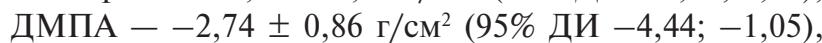

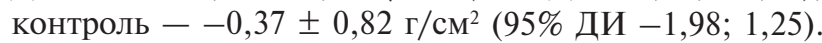
За 24 мес. наблюдения достоверных различий показателей МПКТ у пациенток, получающих ОК, и контролем не выявлено, однако установлено достоверно снижение показателей МПКТ на 5,7 \% в группе, получавшей ДМПА, по сравнению с контролем, с 3,2 \% потерей между 12 и 24 мес. У женщин, принимающих дезогестрел, отмечено снижение МПКТ на 2,6 \% за 24 мес. Изменения МПКТ в группе, получавшей норэтидрон, были недостоверными по сравнению с контролем. Изменения МПКТ у женщин, принимающих ДМПА, за 24 мес. наблюдения были достоверно выше по сравнению с динамикой показателей в остальных группах.

Таким образом, большинство существующих исследований подтверждают положительный эффект ГК на показатели МПКТ и биохимические маркеры костного ремоделирования. Наличие отрицательного влияния ГК на состояние костной ткани или же отсутствие какого-либо эффекта может быть связано с видом или способом введения контрацептива, что требует проведения дальнейших исследований.

\section{Влияние гормональной контрацепции на состояние костной ткани женщин в пременопаузальном периоде}

При анализе влияния ОК на показатели костной ткани у пременопаузальных женщин их положительное влияние показано как в рандомизированных ( $\mathrm{Vol}$ pe A. et al., 1997), так и в проспективных (Shargil A.A., 1985; Gambacciani M. et al., 1994; 2000) и одномоментных исследованиях (Enzelsberger H. et al., 1988; Tuppurainen M. et al., 1994; Masaryk P. et al., 1998). Однако существуют исследования, которые ставят под сомнение положительный эффект ОК на показатели МПКТ (Volpe A. et al., 1993; Fortney J.A. et al., 1994; Beksinska M.E. et al., 2005). Исследований, свидетельствующих об отрицательном эффекте ОК на состояние костной ткани у женщин в перименопаузе, в доступной литературе нами не выявлено.

В единственном обнаруженном 36-месячном рандомизированном сравнительном исследовании влияния ОК на состояние костной ткани у 17 перименопаузальных женщин в возрасте 46-53 лет (Volpe A. et al., 1997) продемонстрировано недостоверное увеличение показателей МПКТ поясничного отдела позвоночника в группе, принимающей ОК, тогда как в контрольной группе установлено достоверное снижение показателей МПКТ.

Положительный эффект ОК продемонстрирован в одномоментном мультицентровом исследовании 2038 женщин (98 пери-, 1940 постменопаузальных), участвовавших в исследовании EVOS (Masaryk P. et al., 1998), 18,3 \% из которых принимали ОК когда-либо в прошлом. У пациенток, принимавших ОК, отмече-

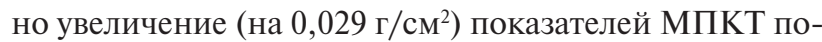

ясничного отдела позвоночника по сравнению с показателями женщин, никогда не принимавших ГК.

В другом одномоментном исследовании (Tuppurainen M. et al., 1994) при обследовании 3222 перименопаузальных женщин (средний возраст - 53,4 года; $29 \%$ когда-либо в прошлом принимали ОК, содержащие 50 мг и меньше ЭЭ; 7,4 \% принимали ОК более 6 лет) установлены слабо выраженные, однако статистически достоверные различия показателей МПКТ поясничного отдела позвоночника у пациенток, принимавших ОK, по сравнению с женщинами, никогда их не принимавшими (соответственно $1,13 \pm 0,16$ и $1,12 \pm 0,16$ г $\left./ \mathrm{cm}^{2}, \mathrm{p}=0,014\right)$. В одномоментном исследовании Enzelsberger H. et al. (1988) также показано, что прием ОК более 10 лет ассоциирован с увеличением МПКТ.

Влияние приема ОК на состояние костной ткани изучено также в одномоментном исследовании в параллельных группах (Ulrich C.M. et al., 1996) у 25 пожилых женщин (средний возраст - 72 года) и 25 их дочерей, находившихся в пременопаузальном периоде (средний возраст - 41 год). В данном исследовании не установлено достоверных различий показателей МПКТ аксиального и периферического скелета у пациенток в зависимости от факта приема ГК. Показатели МПКТ аксиального и всего скелета у пожилых женщин положительно коррелировали с уровнем приема кальция после 60 лет, массой тела, приемом заместительной гормональной терапии на момент исследования и фактом приема ОК в прошлом, а МПКТ аксиального скелета положительно коррелировала с показателями массы тела и приемом ОK в анамнезе.

Однако некоторые исследования свидетельствуют о слабом, недостоверном влиянии ОК на состояние костной ткани у женщин в перименопаузальном периоде. Так, в исследовании Fortney J.A. et al. (1994) при обследовании 352 перименопаузальных женщин в возрасте 40-54 лет, разделенных на 2 группы: принимавшие когда-либо ОК и никогда не принимавшие ОK, установлено статистически недостоверное увеличение показателей МПКТ позвоночника у пациенток, принимающих ОК с более длительным приемом и принимающих ОК в недавнем прошлом, по сравнению с женщинами, никогда не принимавшими ОК. Отсутствие положительного влияния ОК подтверждают и другие исследователи (Beksinska M.E. et al., 2005).

Результаты большинства существующих проспективных исследований все же подтверждают факт положительного влияния ОК на состояние костной ткани у женщин в перименопаузе. В 36-месячном проспективном исследовании 200 перименопаузальных женщин в возрасте 41-49 лет, принимающих 3-фазный ОК, содержащий ЭЭ и левоноргестрел (Shargil A.A., 1985), достоверных изменений показателей МПКТ поясничного отдела позвоночника у пациенток, принимающих ОК, не выявлено, а в контроле отмечено снижение МПКТ позвоночника на $6 \%$, а в 24-месячном проспективном исследовании 37 перименопаузальных женщин в возрасте 45-48 лет, получающих $20 \mu$ ЭЭ 
и $150 \mu$ дезогестрела (Volpe A. et al., 1993), также установлено статистически недостоверное увеличение показателей МПКТ поясничного отдела позвоночника на $8 \%$.

Значительный интерес представляет серия 24-месячных проспективных кальцийконтролируемых исследований, проведенных Gambacciani M. При исследовании 32 перименопаузальных женщин с олигоменореей, получающих $30 \mu$ ЭЭ и $75 \mu$ гестодена или 500 мг ионизированного кальция (Gambacciani M. et al., 1994), установлено достоверное увеличение показателей МПКТ лучевой кости у пациенток, принимающих OK, по сравнению с группой, принимающей препарат кальция. У женщин с олигоменореей, несмотря на дополнительный прием кальция (500 мг/д), на фоне достоверного $(\mathrm{p}<0,001)$ увеличения уровня биохимических маркеров костного ремоделирования отмечено достоверное ухудшение $(-3,4 \%$ за 24 мес., $\mathrm{p}<0,001)$ показателей МПКТ, тогда как у женщин, принимающих низкодозированные ОК, выявлено достоверное снижение биохимических показателей костного метаболизма и незначительно выраженное, однако достоверное увеличение МПКТ $(+1,71 \%, \mathrm{p}<0,01)$.

В другом 24-месячном проспективном кальцийконтролируемом исследовании (Gambacciani M. et al., 1994) с участием 90 перименопаузальных (27 с нормальным менструальным циклом, 54 с олигоменореей) женщин, принимающих ОK, содержащий $20 \mu$ ЭЭ и 0,15 мг дезогестрела или препараты кальция, также показано увеличение показателей МПКТ поясничного отдела позвоночника у пациенток, принимающих ОК, и их снижение у женщин, принимающих кальций. У женщин с олигоменореей, принимающих препараты кальция, установлено снижение уровня гидроксипролина в моче и остеокальцина в сыворотке крови параллельно с достоверным снижением МПКТ позвоночника ( $<<0,0001)$, тогда как у пациенток, принимающих ОK, на фоне отсутствия достоверной динамики уровня остеокальцина отмечено снижение показателей суточной экскреции гидроксипролина на фоне достоверного $(\mathrm{p}<0,001)$ увеличения МПКТ поясничного отдела позвоночника.

Еще одно 24-месячное проспективное кальцийконтролируемое исследование, изучающее эффективность дезогестрелсодержащего ОК ( Gambacciani M. et al., 2000) у 55 здоровых перименопаузальных (18 c нормальным менструальным циклом, 37 с олигоменореей) женщин, получавших $20 \mu$ ЭЭ и 0,15 мг дезогестрела, показало, что у пациенток, принимающих OK, отмечено достоверное увеличение показателей МПКТ шейки бедренной кости по сравнению с исходными данными и их снижение в шейке бедренной кости, треугольнике Варда и трохантере у женщин, принимающих препараты кальция. У женщин с сохраненным менструальным циклом не отмечено изменений в его длительности, уровне гипоталамо-гипофизарных гормонов, биохимических маркеров костного ремоделирования и МПКТ бедренной кости. Установлено, что у женщин с олигоменореей, принимающих только препараты кальция, отмечается увеличение длитель- ности менструального цикла со снижением уровня эстрадиола и увеличением уровня фолликулостимулирующего гормона в сыворотке крови $(\mathrm{p}<0,05)$, а также достоверное увеличение суточной экскреции гидроксипролина и уровня остеокальцина в сыворотке крови на фоне достоверного снижения показателей МПКТ бедренной кости $(\mathrm{p}<0,05)$. У женщин, принимающих OK, уровни остеокальцина в крови и суточной экскреции гидроксипролина достоверно снижались $(\mathrm{p}<0,05)$ на фоне достоверного увеличения $(\mathrm{p}<0,05)$ МПКТ бедренной кости. Таким образом, авторами сделан вывод, что прием ОК в перименопаузальном периоде может способствовать предотвращению увеличения темпов костного метаболизма и увеличивать МПКТ бедренной кости, влияя на регуляцию оварио-менструальной функции женщины.

Существует мнение, что назначение ДМПА в пременопаузальном периоде связано с низкими показателями МПКТ у женщин в постменопаузе, поскольку даже при отмене препарата до наступления менопаузы МПКТ не может самостоятельно восстановиться в этом возрастном периоде, для которго характерна хотя и медленная, но все же прогрессирующая потеря костной массы.

Одномоментные и проспективные исследования, проведенные у женщин в пременопаузальном периоде, показали, что использование ДМПА связано с уменьшением МПКТ, которое, однако, нивелируется после прекращения приема препарата.

При обследовании 346 постменопаузальных женщин (Orr-Walker B.J. et al., 1998), часть из которых в пременопаузе принимали ДМПА (средний возраст начала приема - 41 год, средняя длительность приема 3 года), не обнаружено достоверных отличий МПКТ во всех измеряемых регионах с помощью ДРА у пациенток, принимающих ДМПА, по сравнению с контролем. Не выявлено связи между МПКТ и длительностью приема ДМПА, возрастом прекращения приема или временем, прошедшим с момента прекращения приема ДМПА и наступлением менопаузы.

Таким образом, большинство исследователей придерживаются мнения о том, что прием ГК в перименопаузе, особенно у женщин с олигоменореей, оказывает положительное влияние на темпы потери костной ткани и показатели МПКТ. Однако при назначении этой группы препаратов в перименопаузе следует учитывать их влияние на липидный и углеводный обмены, систему коагуляции и фибринолиза, что требует индивидуализированного подхода при их назначении.

\section{Влияние гормональной контрацепции на состояние костной ткани у женцин с нарушениями оварио-менструального цикла и нарушениями пищевого поведения}

Согласно существующим в настоящее время классификациям длительность нормального менструального цикла составляет 25-34 дня. При ее увеличении более 35 дней регистрируется олигоменорея, а при отсутствии менструации в течение 3 и более месяцев 
(после предшествующего регулярного менструального цикла) - вторичная аменорея.

В настоящее время существует достаточно исследований, изучающих влияние ГК на состояние костной ткани у женщин с олиго- и аменореями различного генеза. Большинство из них, в том числе рандомизированные (Hergenroeder A.C. et al., 1997; CasteloBranco C. et al., 2001) и проспективные (De Crée C. et al., 1988; Gulekli B. et al., 1994; Haenggi W. et al., 1994; Cumming D.C., 1996; Rickenlund A. et al., 2004) свидетельствуют об их положительном влиянии на показатели МПКТ, а также биохимические маркеры костного ремоделирования (Grinspoon S.K. et al., 2003). Однако при анализе литературы выявлено одно рандомизированное (Gibson J.H., 1999) и одно проспективное сравнительное исследование (Gremion G. et al., 2001), свидетельствующие об отсутствии подобного влияния. Исследований, демонстрирующих отрицательный эффект ГК на состояние костной ткани у женщин с аменореями, в доступной литературе не выявлено.

Во всех исследованиях при сравнении МПКТ женщин с олиго- или аменореей и здоровых пациенток установлено, что показатели МПКТ у женщин с аменореей были достоверно ниже (Gulekli B. et al., 1994; Haenggi W. et al., 1994; Cumming D.C., 1996; Rickenlund A. et al., 2004).

В 12-месячном рандомизированном плацебоконтролируемом исследовании (Hergenroeder A.C. et $a l ., 1997)$ у 24 женщин с аменореей гипоталамического происхождения в возрасте 14-28 лет изучено состояние костной ткани в зависимости от вида получаемого контрацептива. Пациентки поучали ОК, содержащий $35 \mu$ ЭЭ и 0,5-1 мг норэтидрона либо 10 мг медроксипрогестерона или плацебо. Установлено, что показатели МПКТ поясничного отдела позвоночника и всего скелета в группе, принимающей ОК, были достоверно выше по сравнению с результатами в группе, принимающей медроксипрогестерон и плацебо, однако МПКТ шейки бедренной кости достоверно не отличались во всех трех группах. Через 12 мес. исследования в группе, принимающей медроксипрогестерон, не выявлено достоверных изменений МПКТ во всех измеряемых регионах скелета.

В другом 12-месячном рандомизированном исследовании (Castelo-Branco C. et al., 2001) с участием 64 женщин с олигоменореей гипоталамического происхождения в возрасте 19-35 лет, разделенных на три группы в зависимости от вида лечения (30 $\mu$ ГЭ + $+0,15$ мг дезогестрела, $20 \mu$ ЭЭ + 0,15 мг дезогестрела и контроль), выявлено увеличение МПКТ поясничного отдела позвоночника в обеих группах, принимающих ОK (I - 2,4 \%; II - 2,5 \%), и снижение показателей в контрольной группе $(1,2 \%, \mathrm{p}<0,05)$. Уровни кальция, фосфатов и остеокальцина в сыворотке крови достоверно снижались в группах, получавших ОК, тогда как в контрольной группе динамика показателей через 12 мес. наблюдения не выявлена.

В мультицентровом рандомизированном двойном слепом плацебо-контролируемом 3-месячном исследовании, проведенном с участием 45 женщин с гипо- таламической аменореей в возрасте 18-40 лет (Grinspoon S.K. et al., 2003), обследуемые были разделены на следующие группы: женщины, принимающие 3-фазный OK, содержащий $35 \mu$ ЭЭ и норгестимата, или плацебо. В группе пациенток, принимающих OK, по сравнению с плацебо установлено достоверно более выраженное снижение уровня $\mathrm{N}$-телопептида $(\mathrm{M} \pm \mathrm{SD}:-13,4 \pm 13,4$ и 1,2 $\pm 23,8$ нмоль эквивалент костного коллагена/моль креатинина, $\mathrm{p}=0,001)$ и Дпир. (-1,2 $\pm 2,9$ и $-0,5 \pm 1,5$ нмоль/моль креатинина, $\mathrm{p}=0,02)$, снижение уровня костноспецифической ЩФ (-5,1 $\pm 3,5$ и -0,4 $\pm 3,1$ нг/мл, p < 0,001), остеокальцина $(-5,9 \pm 3,6$ и $-2,9 \pm 3,7$ нг/мл, $\mathrm{p}=0,016)$ и пропептида проколлагена I типа $(-35,2 \pm 44,6$ и $-0,2 \pm 30,0$ нг/мл, $\mathrm{p}=0,025)$.

Однако в 18-месячном рандомизированном исследовании 34 женщин-атлетов с олиго- и аменореей (Gibson J.H., 1999), получавших эстрогены (1 мг эстриола +2 мг эстрадиола (1-12-й день), 1 мг эстриола +2 мг эстрадиола +1 мг норэтистерона ацетата (13-22-й день), 0,5 мг эстриола + 1 мг эстрадиола (23-28-й день) + 1000 мг кальция карбоната либо 1000 мг кальция карбоната) или не получавших какого-либо лечения (контроль), установлено статистически недостоверное увеличение показателей МПКТ по сравнению с исходными в группе женщин, принимающих эстрогены.

В 12-месячном рандомизированном плацебо-контролируемом исследовании (Hergenroeder A.C. et al., 1997) 24 женщины с аменореей гипоталамического происхождения в возрасте 14-28 лет проведено сравнение влияния 10 мг медроксипрогестерона ацетата и других ОК по сравнению с контролем на состояние костной ткани. Показано, что показатели МПКТ поясничного отдела позвоночника и всего скелета в группе, принимающей ОК, были достоверно выше по сравнению с результатами в группе медроксипрогестерона ацетата и плацебо, а показатели МПКТ шейки бедренной кости достоверно не отличались во всех группах. Однако за время наблюдения установлено отсутствие достоверных изменений во всех измеряемых регионах в группе, принимающей медроксипрогестерон.

В табл. 5 представлены результаты существующих проспективных исследований, изучавших влияние ОК на состояние костной ткани у женщин с олиго-/аменореей.

Anorexia nervosa (AH) - нарушение пищевого поведения, обычно манифестирующееся в подростковом и юношеском возрасте и характеризующееся чрезмерным увлечением различными диетами, ведущими к потере массы тела, часто сопровождающееся чрезмерными физическими упражнениями, усиленным желанием потери массы тела и боязнью ожирения. У части пациентов периоды голодания чередуются с периодами нормального питания. Риск развития этого заболевания среди молодых женщин составляет от 0,3 до $1 \%$, при этом отмечается большая частота доклинической АН.

В настоящее время существуют противоречивые сведения о влиянии ГК на состояние костной ткани у женщин с АН. Большинство исследований свидетель- 
ствуют об отсутствии какого-либо эффекта ГК на состояние костной ткани у пациенток с АН, в том числе и в рандомизированные исследования (Klibanski A. et al., 1995; Gordon C.M. et al., 2002; Muñoz M.T. et al., 2002; Golden N.H. et al., 2002; Grinspoon S. et al., 2002), и только некоторые из них подтверждают положительное влияние ГК на состояние костной ткани (Seeman E. et al., 1992; Karlsson M.K. et al., 2000).

Так, в одномоментном исследовании (Seeman E. et al., 1992) при участии 117 женщин (с первичной и вторичной аменореей, а также здоровых пациенток (контроль)), разделенных на группы в зависимости от приема ОК, установлено, что исходные показатели МПКТ здоровых женщин были достоверно выше по сравнению с соответствующими у женщин с АН. МПКТ поясничного отдела позвоночника у пациенток, принимающих ОК в течение $31,8 \pm 8,3$ мес., была достоверно выше по сравнению с женщинами, не принимающими их (соответственно $1,14 \pm 0,05$ и
$1,02 \pm 0,02$ г $\left./ \mathrm{cm}^{2}, \mathrm{p}<0,02\right)$, но ниже, чем в контроле

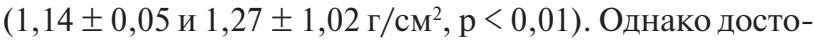
верной динамики показателей МПКТ шейки бедренной кости под влиянием приема ОК не выявлено.

В другом одномоментном исследовании (Karlsson M.K. et al., 2000) при обследовании 366 женщин (c АН, принимающих и не принимающих ОK, а также пациенток с диагнозом АН в прошлом и здоровых женщин (контроль)) также показано, что показатели МПКТ здоровых пациенток были достоверно выше по сравнению с соответствующими у женщин с АН. Снижение показателей МПКТ позвоночника и бедренной кости у женщин с АН, не принимающих ОК, co-

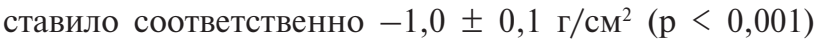
и $-0,3 \pm 0,1 г / \mathrm{cm}^{2}(\mathrm{p}<0,05)$. Снижение показателей МПКТ у женщин с АН, принимающих ОК, было менее выражено и составило в поясничном отделе позвоночника $-0,6 \pm 0,1 \mathrm{SD}(\mathrm{p}<0,001)$, но не в шейке бе-

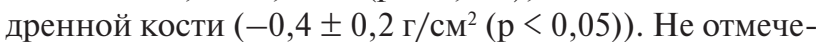

Таблица 5. Проспективные исследования, изучающие эффект гормональных контрацептивов на показатели костной ткани у женщин с олиго- и аменореей

\begin{tabular}{|c|c|c|}
\hline Авторы & Дизайн исследования & Результаты \\
\hline $\begin{array}{l}\text { De Creé C. } \\
\text { et al., } 1988\end{array}$ & $\begin{array}{l}\text { 8-мес. проспективное исследование. } 11 \text { женщин-спорт- } \\
\text { сменок с нарушением МЦ в возрасте } 18-29 \text { лет. } 2 \text { груп- } \\
\text { пы: } 50 \text { цг ЭЭ + } 2 \text { мг ципротерона ацетата }(n=7), \text { контроль } \\
\text { (n = 4). МИ: ДРА (поясничный отдел позвоночника, ОФА (лу- } \\
\text { чевая кость) }\end{array}$ & $\begin{array}{l}\text { Увеличение показателей МПКТ поясничного отдела позвоноч- } \\
\text { ника (M } \pm \text { SD) на 9,50 } \pm 2,45 \text { \% в группе, принимавшей ОК, } \\
\text { тогда как показатели кортикальной КТ лучевой кости досто- } \\
\text { верно не изменялись }\end{array}$ \\
\hline $\begin{array}{l}\text { Gulekli B. } \\
\text { et al., } 1994\end{array}$ & $\begin{array}{l}\text { 36-мес. проспективное исследование. } 85 \text { женщин с аме- } \\
\text { нореей в прошлом }(\mathrm{n}=33) \text { или настоящем }(\mathrm{n}=52) \text { в } \\
\text { возрасте } 17-40 \text { лет. Средняя длительность аменореи } \\
46,5 \text { мес. }(8 \text { мес. }-21 \text { год). Женщины, получавшие синте- } \\
\text { тические эстрогены (10-50 нг ЭЭ) }(\mathrm{n}=40), \text { натуральные } \\
\text { эстрогены (премарин или эстрадиола валерат) }(\mathrm{n}=10), \\
50 \text { мг трансдермального эстрадиола }(\mathrm{n}=8), \text { бромкриптин } \\
(\mathrm{n}=9) \text {, диетотерапию, направленную на прибавку массы } \\
\text { тела }(\mathrm{n}=6), \text { контроль }(\mathrm{n}=12) . \text { МИ: ДРА (поясничный отдел } \\
\text { позвоночника) }\end{array}$ & 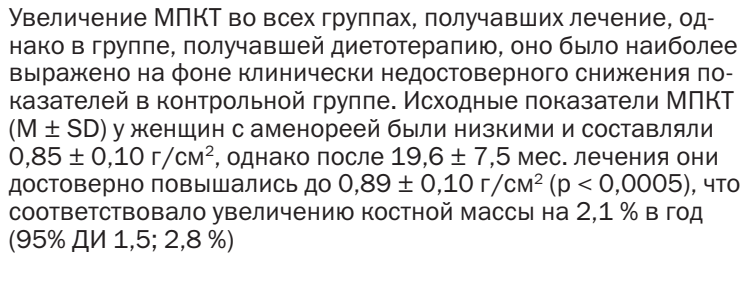 \\
\hline $\begin{array}{l}\text { Haenggi W. } \\
\text { et al., } 1994\end{array}$ & $\begin{array}{l}\text { 24-мес. проспективное исследование. } 21 \text { женщина с аме- } \\
\text { нореей гипоталамического или яичникового происхожде- } \\
\text { ния, } 123 \text { здоровые женщины (контроль) в возрасте 18- } \\
45 \text { лет. } 2 \text { группы: } 30 \mu \text { Эг + } 0,15 \text { мг дезогестрела }(\mathrm{n}=15) \text {, } \\
\text { контроль (n = 123). МИ: ДРА (поясничный отдел позвоноч- } \\
\text { ника, проксимальный отдел бедренной кости) }\end{array}$ & $\begin{array}{l}\text { Исходные показатели МПКТ были ниже у женщин с амено- } \\
\text { реей по сравнению со здоровыми пациентками и составля- } \\
\text { ли } 85 \text { \% ( }<\text { < 0,0001) от показателя контроля в позвоночни- } \\
\text { ке, } 92 \%(p<0,02) \text { в шейке бедренной кости, } 90 \%(p<0,03) \text { в } \\
\text { треугольнике Варда, } 92 \%(p<0,0001) \text { в диафизе большебер- } \\
\text { цовой кости и } 92 \%(p<0,03) \text { в ее эпифизе. У женщин, прини- } \\
\text { мающих ОК, установлено достоверное увеличение МПКТ (от } \\
0,2 \text { до 2,9\%) в поясничном отделе позвоночника ( }<<0,0012) \text {, } \\
\text { треугольнике Варда ( }<<0,033)\end{array}$ \\
\hline $\begin{array}{l}\text { Cumming D.C., } \\
1996\end{array}$ & $\begin{array}{l}\text { 24-мес. проспективное исследование. } 13 \text { женщин-бегуний } \\
\text { с аменореей в возрасте } 23-34 \text { лет. } 3 \text { группы: 0,0625 мг } \\
\text { конъюгированных эстрогенов }(n=6), 50 \text { нг трансдермаль- } \\
\text { ного эстрадиола }(n=2), \text { контроль }(n=5) \text { МИ: ДРА (поя- } \\
\text { сничный отдел позвоночника, шейка бедренной кости, тре- } \\
\text { угольник Варда) }\end{array}$ & $\begin{array}{l}\text { Достоверное увеличение МПКТ (M } \pm \text { SEM) позвоночника } \\
(8,0 \pm 1,2 \%) \text { и шейки бедренной кости }(4,1 \pm 0,3 \%) \text { в группе, } \\
\text { получающей эстрогены. Статистически недостоверное (менее } \\
2,5 \%) \text { уменьшение МПКТ позвоночника и бедренной кости в } \\
\text { контрольной группе }\end{array}$ \\
\hline $\begin{array}{l}\text { Rickenlund A. } \\
\text { et al., } 2004\end{array}$ & $\begin{array}{l}\text { 10-мес. проспективное исследование. } 38 \text { женщин (26 ат- } \\
\text { леток: } 13 \text { с нормальным МЦ, } 13 \text { с олигоменореей), } 12 \\
\text { женщин, не занимающихся спортом, с нормальным МЦ в } \\
\text { возрасте 16-35 лет. Каждая группа получала } 30 \text { нг ЭЭ + } \\
150 \text { нг левоноргестрела. МИ: ДРА (поясничный отдел по- } \\
\text { звоночника, весь скелет) }\end{array}$ & 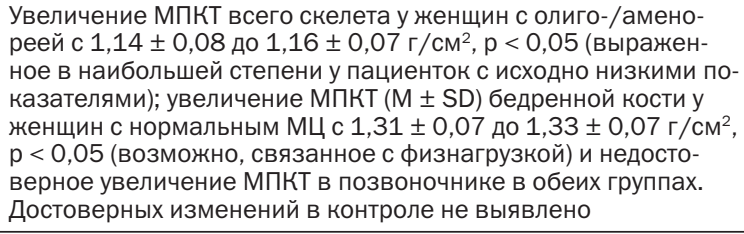 \\
\hline $\begin{array}{l}\text { Gremion G. } \\
\text { et al., } 2001\end{array}$ & $\begin{array}{l}\text { 12-мес. проспективное исследование. } 30 \text { женщин (бегуньи } \\
\text { на длинные дистанции) в возрасте 19-37 лет. Женщины: } \\
19-\text { принимающие ОК, } 10-\text { не принимающие ОК с нор- } \\
\text { мальным МЦ, } 11 \text { - не принимающие ОК с олиго- и амено- } \\
\text { реей более } 12 \text { мес. МИ: ДРА (поясничный отдел позвоноч- } \\
\text { ника, проксимальный отдел бедренной кости, середина бе- } \\
\text { дренной кости), БХМ (остеокальцин) }\end{array}$ & 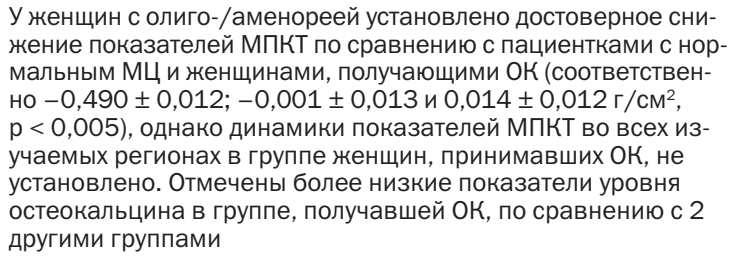 \\
\hline
\end{tabular}

Примечание: сокращения те же, что в табл. 4. 
но достоверного снижения показателей МПКТ позвоночника и шейки бедренной кости у женщин с диагнозом АН в анамнезе $(-0,3 \pm 0,2 \mathrm{SD})$. У пациенток с АH, не принимающих ОК, МПКТ позвоночника и шейки бедренной кости составила $-1,6 \pm 0,1$ и $-1,1 \pm 0,1 \mathrm{SD}$ $(\mathrm{p}<0,001)$. Менее выраженное снижение отмечено в группе, получавшей эстрогены $(-1,2 \pm 0,2$ и $-0,6 \pm 0,2$ $\mathrm{SD}(\mathrm{p}<0,001))$, а наименьшее снижение показателей установлено у женщин с диагнозом АН в анамнезе $(-0,6 \pm 0,1(\mathrm{p}<0,01)$ и $-0,5 \pm 0,2 \mathrm{SD}(\mathrm{p}<0,05))$.

Существующие на сегодняшний день рандомизированные исследования, изучавшие влияние ГК на состояние костной ткани у женщин с АН, свидетельствуют об отсутствии какого-либо эффекта. В 18-месячном рандомизированном проспективном исследовании с участием 48 женщин в возрасте 16-42 лет с АН, разделенных на три группы (0,625 мг премарина и 5 мг провера, 35 нг ЭЭ, контроль) (Klibanski A. et al., 1995), не обнаружено достоверных изменений МПКТ в группе, получавшей эстрогены, по сравнению с показателями контроля, однако выявлено повышение показателей МПКТ на 4 \% в группе женщин с исходно низкими показателями массы тела (менее 70 \% от оптимальной), получавших эстрогены. Тогда как у пациенток контрольной группы с исходно низкими показателями массы тела (менее 70 \%) отмечено, напротив, $20,1 \%$ снижение МПКТ. У женщин контрольной группы с самостоятельным восстановлением менструальной функции (все имели исходные показатели массы тела больше $70 \%$ от оптимальной) отмечено повышение показателей МПКТ на 19,3\%.

В другом 12-месячном рандомизированном проспективном сравнительном исследовании 51 женщины в возрасте 14-28 лет с АН, принимавших ОК, содержащий $20 \mu$ Г ЭЭ и 0,1 мг левоноргестрела, или 50 мг дегидроэпиандростерона (Gordon C.M. et al., 2002), установлено достоверное увеличение МПКТ всего скелета $(+1,7 \%)$, недостоверное увеличение МПКТ позвоночника и снижение экскреции $\mathrm{N}$-телопептида $(\mathrm{p}<0,05)$ с мочой у пациенток обеих групп, а также транзиторное достоверное увеличение костноспецифической ЩФ и остеокальцина (маркеров формирования костной ткани) на 6-й и 9-й месяц у женщин первой группы.

Влияние ГК изучено еще в одном 9-месячном рандомизированном проспективном исследовании с участием 60 женщин с АН. Пациентки были разделены на четыре группы: женщины, принимающие ОК, содержащий $35 \mu$ ЭЭ и 0,4 мг норэтиндрона; пациентки, принимающие $30 \mu$ г/кг рекомбинантного человеческого инсулиноподобного фактора роста I (РЧИПФР-I),

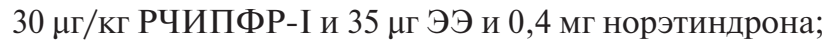
а также контроль (плацебо РЧИПФР-І, без ОК) (Grinspoon S. et al., 2002). Достоверного влияния ОК на показатели МПКТ во всех регионах, исследуемых с помощью ДРА, авторами не выявлено.

В 36-месячном проспективном исследовании 50 женщин с АН в возрасте 13-21 года (Golden N.H. et al., 2002), получавших трехфазный ОК (35 $\mu$ ГЭ И норгестимат), монофазные ОК (35 $\mu$ Г ЭЭ и 0,25 мг нор- гестимата, $30 \mu г$ ЭЭ и 0,3 мг норгестрела, $30 \mu г$ ЭЭ и 1,5 мг норэтиндрона, $30 \mu$ ЭЭ и 0,15 мг левоноргестрела, 20 гг ЭЭ и 1 мг левоноргестрела) или не принимавших ОК (контроль), показано, что исходные показатели МПКТ у пациенток с АН были снижены по сравнению с референтной базой молодых женщин. При анализе динамики показателей МПКТ после приема ОК авторами не получено достоверных изменений МПКТ поясничного отдела позвоночника и шейки бедренной кости по сравнению с исходными в какой-либо из групп, получающих ОК, или в контрольной группе. Еще одно 12-месячное проспективное исследование женщин, принимающих ОК, содержащий 50 нг ЭЭ и 0,5 мг норгестрела (Muñoz M.T. et al., 2002), достоверных изменений показателей МПКТ поясничного отдела позвоночника по сравнению с исходными показателями не подтвердило.

\section{Гормональная контрацепция и риск остеопоротических переломов}

Немногочисленными, однако также противоречивыми являются литературные данные, касающиеся риска переломов у пациенток, принимающих препараты гормональной контрацепции. В проспективном исследовании Cooper C. et al. (1993) впервые изучена связь между приемом ОК и риском развития переломов среди 46000 женщин, принимавших участие в Royal College of General Practitioners Oral Contraception Study в течение 482083 человеко-лет наблюдения. В данном исследовании показано, что риск переломов был ниже у многорожавших, некурящих женщин, а также обследованных с низкими социально-экономическими условиями жизни. С учетом данных факторов установлено, что женщины, принимавшие когда-либо таблетированные OK, имели достоверно более высокий риск последующих переломов по сравнению с обследованными, не принимавшими их (OP = 1,20, 95\% ДИ 1,08-1,34). Однако при анализе частоты переломов предплечья достоверного влияния ОК не установлено.

Подобные результаты получены в другом проспективном исследовании, проведенном Vessey M. et al. (1998), в котором изучена частота переломов различной локализации у 17032 женщин, принимавших участие в Oxford-Family Planning Association contraceptive study с 1968 по 1994 год (310 000 человеко-лет). Среди обследованных у 1308 зарегистрирован хотя бы один перелом за период наблюдения. Наиболее частыми были переломы лучевой, локтевой костей, тарзальной и метатарзальной кости и лодыжки. При анализе всех переломов установлено достоверное увеличение $(\mathrm{p}=0,001)$ их риска при увеличении длительности приема ОК. Наиболее высокий риск развития переломов $(1,3 ; 95 \%$ ДИ $1,1-1,5)$ установлен у женщин, принимающих ОК на момент обследования или принимавших ОК в недавнем прошлом. Отмечено $20 \%$ увеличение риска переломов в целом у женщин, когда-либо принимающих ОК, по сравнению с женщинами, никогда не принимавшими ОК.

В исследовании Vestergaard P. et al. (2006) принимали участие 64548 женщин, получивших перело- 
мы в 2000 году. Пациентки принимали ОК с 1996 до 2000 г. Кроме того, в данном исследовании участвовали 193641 пациентка без переломов в анамнезе. Предварительный анализ результатов показал, что использование ОК в низких дозах ассоциировано со слабым увеличением риска переломов. Однако при стандартизации групп по ряду факторов (прием каких-либо других медикаментов, количество беременностей, предшествующие переломы, другие заболевания и ряд социальных факторов) не обнаружено увеличения риска переломов ни в одной возрастной группе, а также независимо от дозы ОК.

Michaelsson K. et al. (1999) проведено популяционное (почтовый или телефонный опросы) исследование «случай - контроль» по изучению частоты переломов бедренной кости у шведских постменопаузальных женщин в возрасте 50-81 года. Среди обследованных женщин $130(11,6 \%)$ в основной группе и $562(19,0 \%)$ в контрольной принимали когда-либо ОК, прием которых был ассоциирован с $25 \%$ снижением частоты переломов бедренной кости (ОР $=0,75 ; 95 \%$ ДИ 0,590,96). У пациенток, принимавших ОК в высоких дозах (эквивалент $\geq 50$ мкг ЭЭ в таблетке), риск развития переломов бедренной кости был на $44 \%$ ниже по сравнению с женщинами, которые никогда не принимали OK $(0,56 ; 95 \%$ ДИ 0,42-0,75). В данном исследовании не выявлено различий в частоте переломов в зависимости от длительности приема ОК и времени с момента окончания приема, однако при сравнении показателей с данными пациенток, не принимавших никогда OK, риск развития переломов был ниже в зависимости от возраста: $\mathrm{OP}=0,69(0,51-0,94)$ для пациенток, принимающих ОК после 40 лет, 0,82 $(0,57-1,16)$ для женщин, принимающих ОК в возрасте 30-39 лет, и 1,26 (0,76-2,09) для женщин моложе 30 лет.

20-21 июня 2005 года в Женеве ВО3 проведено согласительное совещание, оценивающее существующие сведения относительно связи между гормональной контрацепцией и состоянием костной ткани, в результате которого появились рекомендации ВОЗ в отношении безопасности применения различных групп ГК в отношении костной ткани (www.who.int/ reproductive-health). Основные положения данного документа изложены ниже.

\section{Комбинированные методы контрацепции}

1. Использование комбинированных ОК обладает небольшим, клинически недостоверным влиянием на костную ткань.

2. Подростки, использующие ОК, могут иметь несколько меньшие показатели МПКТ, чем их сверстницы, не использующие ОК, тогда как у перименопаузальных женщин, использующих ОK, показатели МПКТ несколько выше по сравнению с женщинами, не использующими ОК.

3. Количество исследований, изучавших частоту переломов у женщин, принимающих ОК, недостаточно и противоречиво. Данные о влиянии других форм комбинированной контрацепции (инъекционной, накожной, внутривлагалищной и др.) недостаточны или не существуют вообще.

\section{Гестагенные контрацептивы}

1. Имплантаты, содержащие левоноргестрел, не обладают отрицательным влиянием на показатели МПКТ.

2. Другие низкодозированные гестагенные контрацептивы (таблетки, другие имплантаты и внутриматочные системы, содержашие левоноргестрел) не оказывают отрицательного действия на МПКТ, хотя данные о их безопасности в отношении костной ткани ограниченны.

3. Использование ДМПА у женщин ведет к гипоэстрогении. Некоторые исследования подтверждают отрицательное влияние ДМПА на показатели МПКТ. Использование ДМПА у женшин, не достигших пика костной массы, задерживает его формирование по сравнению с пациентками, не принимающими ДМПА.

4. Одномоментные исследования выявили снижение МПКТ у женщин, длительно принимающих ДМПА, приблизительно на 0,5 SD в шейке бедренной кости и позвоночнике по сравнению с женщинами, не использующими ДМПА. Лонгитудинальные исследования у молодых женщин с задержкой менархе (больше 18 лет) и подростков с менархе меньше 18 лет выявили 5-7\% потерю костной ткани (приблизительно $0,5 \mathrm{SD})$ в шейке бедренной кости и позвоночнике после 2-летнего постоянного приема ДМПА.

5. Показатели МПКТ при прекращении использования ДМПА восстанавливаются, несмотря на возраст, за исключением женщин в перименопаузальном периоде. За период от 2 до 3 лет у молодых женщин показатели ДМПА после прекращения приема сравнимы с показателями у пациенток, не принимавших его, однако не ясно, достигают ли подростки, принимавшие ДМПА, своего потенциального пика костной массы. Женщины, принимающие ДМПА в перименопаузе, могут не успеть восстановить показатели МПКТ перед вступлением в постменопаузу.

6. Данных относительно влияния ДМПА при приеме в репродуктивном периоде на частоту переломов (в репродуктивном и в дальнейшем в постменопаузальном периоде) недостаточно, однако это влияние маловероятно в связи с обратимостью действия ДМПА на костную ткань.

7. В связи с тем, что данные относительно влияния других инъекционных гестагенных контрацептивов (норэтистерона энантат) отсутствуют, данные относительно ДМПА могут быть применимы к НЭТ-ЭН.

8. ДМПА является высокоэффективным и широкодоступным методом предупреждения беременности, что следует учитывать при выборе метода контрацепции.

В связи с вышеизложенным ВОЗ были даны следующие рекомендации.

Учитывая влияние ГК на метаболизм костной ткани, рекомендовано следующее:

1. Не должно существовать ограничений к использованию ДМПА, включая длительное применение, у женщин в возрасте 18-45 лет, желающих использовать данный метод контрацепции. Среди подростков с показателями возраста менархе более 18 лет и жен- 
щин старше 45 лет использование ДМПА должно быть взвешено в связи с существующим риском переломов. Существуют в настоящее время данные о безопасности длительного назначения ДМПА, в связи с чем необходимо в дальнейшем после проведенных исследований пересмотреть показания к назначению данного метода в этих возрастных группах с учетом рисков и преимуществ при продолжении терапии в индивидуальном порядке.

2. Рекомендации относительно ДМПА касаются в той же степени использования НЭТ-ЭН.

3. Не существует ограничений к использованию других только прогестагенных контрацептивов среди женщин, включая ограничения относительно длительности применения.

4. Не существует ограничений к использованию комбинированных гормональных контрацептивов среди женщин, включая ограничения относительно длительности применения.
В 2007 году основные положения о возможном влиянии контрацептивов на структурно-функциональное состояние костной ткани были пересмотрены (www.who.int/reproductive-health) согласно обновленным данным проведенных исследований. В рекомендациях ВОЗ было отмечено, что женщины в возрасте 18-45 лет могут использовать ДМПА и другие инъекционные прогестинсодержащие ГК без какихлибо ограничений. Подростки и женщины старше 45 лет, использующие ДМПА и другие инъекционные ГК, также могут использовать их, но учитывая данные о возможном снижении показателей МПКТ при их применении. Ни для каких других прогестиновых ГК не существует ограничений к использованию в связи с их действием на состояние костной ткани. Для комбинированных методов контрацепции (при использовании эстрогена и гестагена) при выборе их женщиной не существует каких-либо ограничений в отношении костной ткани.

\section{Список литературы}

1. Afghani A., Abbott A.V., Wiswell R.A. et al. Bone mineral density in Hispanic women: role of aerobic capacity, fatfree mass, and adiposity // Int. J. Sports Med. - 2004 _ - 25. P. 384-390.

2. Allali F., El Mansouri L., Abourazzak F.-Z. et al. The effect of past use of oral contraceptive on bone mineral density, bone biochemical markers and muscle strength in healthy pre and post menopausal women // BMC Womens Health. 2009. - 9. - P. 31-34.

3. Bedford J.L., Barr S.I. The relationship between 24-hr urinary cortisol and bone in healthy young women // Int. J. Behav. Med. - 2010. - 17(3). - P. 207-215.

4. Beksinska M.E., Smit J.A., Kleinschmidt I. et al. Bone mineral density in women aged 40-49 years using depot-medroxyprogesterone acetate, norethisterone enanthate or combined oral contraceptives for contraception // Contraception. -2005 . -71 . - P. 170-175.

5. Berenson A.B., Breitkopf C.R., Grady J.J. et al. Effects of hormonal contraception on bone mineral density after 24 months of use // Obstet. Gynecol. - 2004. - 103. - P. 899-8906.

6. Berenson A.B., Radecki C.M., Grady J.J. et al. A prospective, controlled study of the effects of hormonal contraception on bone mineral density // Obstet. Gynecol. - 2001. - 98. - P. 576-582.

7. Berenson A.B., Rahman M., Breitkopf C.R. Effects of Depot Medroxyprogesterone Acetate and $20 \mu \mathrm{g}$ Oral Contraceptives on Bone Mineral Density // Obstet Gynecol. - 2008. 112(4). - P. 788-799.

8. Burr D.B., Yoshikawa T., Teegarden D. et al. Exercise and oral contraceptive use suppress the normal age-related increase in bone mass and strength of the femoral neck in women 18-31 years of age // Bone. -2000 . -27 . - P. 855-863.

9. Caird L.E., Reid-Thomas V., Hannan W.J. et al. Oral progestogen-only contraception may protect against loss of bone mass in breast-feeding women // Clin. Endocrinol. (Oxf.). 1994. - 41. - P. 739-745.

10. Castelo-Branco C., Martinez de Osaba M.J., Pons R. et al. Effects on bone mass of two oral contraceptives containing ethinylestradiol and cyproterone acetate or desogestrel: results of a 2-year follow-up // Eur. J. Contracept. Reprod. Health Care. $-1998 .-3 .-$ P. $79-84$.

11. Castelo-Branco C., Vicente J.J., Pons F. et al. Bone mineral density in young, hypothalamic oligoamenorrheic women treated with oral contraceptives // J. Reprod. Med. - 2001. 46. $-875-879$.

12. Cobb K.L., Kelsey J.L., Sidney S. et al. Oral contraceptives and bone mineral density in white and black women in CARDIA // Osteoporos. Int. - 2002. - 13. - P. 893-900.
13. Cooper C., Hannaford P., Croft P., Kay C.R. Oral contraceptive pill use and fractures in women: a prospective study // Bone. - 1993. - 14(1). - P. 41-45.

14. Cromer B.A., Bonny A.E., Stager M. et al. Bone Mineral Density in Adolescent Females Using Injectable or Oral Contraceptives: A 24 Month Prospective Study // Fertil. Steril. 2008. - 90(6). - P. 2060-2067.

15. Cromer B.A., Blair J.M., Mahan J.D. et al. A prospective comparison of bone density in adolescent girls receiving depot medroxyprogesterone acetate, levonorgestrel, or oral contraceptives // J. Pediatr. - 1996. - 129. - P. 671-676.

16. Cromer B.A., Stager M., Bonny A. et al. Depot medroxyprogesterone acetate, oral contraceptives and bone mineral density in a cohort of adolescent girls // J. Adolesc. Health. 2004. - 35. - P. 434-441.

17. Cumming D.C. Exercise associated amenorrhea, low bone density, and estrogen replacement therapy // Arch. Intern. Med. - 1996. $-156 .-$ P. 2193-2195.

18. De Crée C., Lewin R., Ostyn M. Suitability of cyproterone acetate in the treatment of osteoporosis associated with athletic amenorrhea // Int. J. Sports Med. - 1988. - 9. P. 187-192.

19. Elgán C., Samsioe G., Dykes A.K. Influence of smoking and oral contraceptives on bone mineral density and bone remodeling in young women: a 2-year study // Contraception. 2003. - 67. - P. 439-447.

20. Endrikat J., Mih E., Dusterberg B. et al. A 3 year doubleblind randomized controlled study on the influence of 2 oral contraceptives containing either $20 \mu \mathrm{g}$ or $30 \mu \mathrm{g}$ ethinylestradiol in combination with levonorgestrel on bone mineral density // Contraception. - 2004. - 69. - P. 179-187

21. Enzelsberger H., Metka M., Heytmanek G. et al. Influence of oral contraceptive use on bone density in climacteric women // Maturitas. - 1988. - 9. - P. 375-378.

22. Fortney J.A., Feldblum P.J., Talmage R.V. et al. Bone mineral density and history of oral contraceptive use // J. Reprod. Med. - 1994. - 39. - P. 105-109.

23. Gambacciani M., Ciaponi M., Cappagli B. et al. Longitudinal evaluation of perimenopausal bone loss: effects of a lowdose oral contraceptive preparation on bone mineral density and metabolism // Osteoporos. Int. - 2000. - 11. - P. 544-548.

24. Gambacciani M., Spinetti A., Cappagli B. et al. Hormone replacement therapy in perimenopausal women with a low dose oral contraceptive preparation: effects on bone mineral density and metabolism // Maturitas. - 1994. - 19. P. 125-131.

25. Gambacciani M., Spinetti A., Taponeco F. et al. Longitudinal evaluation of perimenopausal vertebral bone loss: ef- 
fects of a low-dose oral contraceptive preparation on BMD and metabolism // Obstet. Gynecol. - 1994. - 83. - P. 392-396.

26. Garnero P., Sornay-Rendu E., Delmas P.D. Decreased bone turnover in oral contraceptive users // Bone. - 1995. 16. - P. 499-503.

27. Gbolade B., Ellis S., Murby B. et al. Bone density in long term users of depot medroxyprogesterone acetate // Br. J. Obstet. Gynaecol. - 1998. - 105. - P. 790-794.

28. Gibson J.H. Treatment of reduced bone mineral density in athletic amenorrhea: a pilot study // Osteoporos Int. 1999. - 10. - P. 284-289.

29. Golden N.H., Lanzkowsky L., Schebendach J. et al. The effect of estrogen-progestin treatment on bone mineral density in anorexia nervosa // J. Pediatr. Adolesc. Gynecol. - 2002. 15. - P. 135-143.

30. Goldsmith N.F., Johnston J.O. Bone mineral effects of oral contraceptives, pregnancy and lactation // J. Bone Joint Surg. $-1975 .-57 .-$ P. 657-668.

31. Gordon C.M., Grace E., Emans S.J. et al. Effects of oral dehydroepiandrosterone on bone density in young women with anorexia nervosa: a randomized trial // J. Clin. Endocrinol. Metab. - 2002. - 87. - P. 4935-4941.

32. Gremion G., Rizzoli R., Slosman D. et al. Oligoamenorrheic long-distance runners may lose more bone in позвоночник than in femur // Med. Sci. Sports Exerc. 2001. - 33. - P. 15-21

33. Grinspoon S., Thomas L., Miller K. et al. Effects of recombinant human IGF-I and oral contraceptive administration on bone density in anorexia nervosa // J. Clin. Endocrinol. Metab. - 2002. - 87. - P. 2883-2891.

34. Grinspoon S.K., Friedman A.J., Miller K.K. et al. Effects of a triphasic combination oral contraceptive containing norgestimate/ethinyl estradiol on biochemical markers of bone metabolism in young women with osteopenia secondary to hypothalamic amenorrhea // J. Clin. Endocrinol. Metab. 2003. - 88. - P. 3651-3656.

35. Guilbert E., Black A., Dunn S. et al. Missed hormonal contraceptives: new recommendations // J. Obstet. Gynaecol. Can. - 2008. - 30. - P. 1050-1062.

36. Gulekli B., Davies M.C., Jacobs H.S. Effect of treatment on established osteoporosis in young women with amenorrhea // Clin. Endocrinol. (Oxf.). - 1994. - 41. - P. 275-281.

37. Haenggi W., Casez J.P., Birkhaeuser M.H. et al. Bone mineral density in young women with long-standing amenorrhea: limited effect of hormone replacement therapy with ethinylestradiol and desogestrel // Osteoporos. Int. - 1994. - 4. P. $99-103$

38. Hall M.L., Heavens J., Cullum I.D. et al. The range of bone density in normal British women // Br. J. Radiol. 1990. - 63. - P. 266-269.

39. Hartard M., Bottermann P., Bartenstein P. et al. Effects on bone mineral density of low-dosed oral contraceptives compared to and combined with physical activity // Contraception. $-1997 .-55$. - P. 87-90.

40. Hartard M., Kleinmond C., Kirchbichler A. et al. Age at first oral contraceptive use as a major determinant of vertebral bone mass in female endurance athletes // Bone. - 2004. 35. - P. 836-841.

41. Hawker G.A., Forsmo S., Cadarette S.M. et al. Correlates of forearm bone mineral density in young Norwegian women // Am. J. Epidemiol. - 2002. - 156. - 418-27.

42. Hergenroeder A.C., Smith E.O., Shypailo R. et al. Bone mineral changes in young women with hypothalamic amenorrhea treated with oral contraceptives, medroxyprogesterone, or placebo over 12 months // Am. J. Obstet. Gynecol. - 1997. 176. $-1017-25$

43. Hreshchyshyn M.M., Hopkins A., Zylstra S. et al. Associations of parity, breast-feeding, and birth control pills with lumbar spine and femoral neck bone densities // Am. J. Obstet. Gynecol. - 1988. - 159. - P. $318-322$.

44. Karlsson M.K., Weigall S.J., Duan Y. et al. Bone size and volumetric density in women with anorexia nervosa receiving estrogen replacement therapy and in women recover- ing from anorexia nervosa // J. Clin. Endocrinol. Metab. 2000. - 85. - P. 3177-3182.

45. Kitai E., Blum M., Kaplan B. The bone sparing effect of oral contraceptive use in non-smoking women // Clin. Exp. Obstet. Gynecol. - 1992. - 19. - P. 30-33.

46. Kleerekoper M., Brienza R.S., Schultz L.R. et al. Oral contraceptive use may protect against low bone mass: Henry Ford Hospital Osteoporosis Cooperative Research Group // Arch. Intern. Med. - 1991. - 151. - P. 1971.

47. Klibanski A., Biller B.M., Schoenfeld D.A. et al. The effects of estrogen administration on trabecular bone loss in young women with anorexia nervosa // J. Clin. Endocrinol. Metab. - 1995. - 80. - P. 898-904.

48. Kreipe R.E., Hicks D.G., Rosier R.N. et al. Preliminary findings on the effects of sex hormones on bone metabolism in anorexia nervosa // J. Adolesc. Health. - 1993. - 14. P. 319-324.

49. Kuhl H. Pharmacology of estrogens and progestogens: influence of different routes of administration // Climacteric. -2005 . -8 (Suppl. 1). - P. 3-63

50. Laitinen K., Valimaki M., Keto P. Bone mineral density measured by dual-energy X-ray absorptiometry in healthy Finnish women // Calcif. Tissue Int. - 1991. - 48. - P. 224-231.

51. Lara-Torre E., Edwards C.P., Perlman S. et al. Bone mineral density in adolescent females using depot medroxyprogesterone acetate // J. Pediatr. Adolesc. Gynecol. - 2004. 17. - P. 17-21.

52. Lee J.K., Parisi S.M., Akers A.Y., Borrerro S., Schwarz E.B. The Impact of Contraceptive Counseling in Primary Care on Contraceptive Use // J. Gen. Intern. Med. - 2011. 26(7). - P. 731-736.

53. Lindsay R., Tohme J., Kanders B. The effect of oral contraceptive use on vertebral bone mass in pre- and post-menopausal women // Contraception. - 1986. - 34. - P. 333-340.

54. Lloyd T., Buchanan J.R., Ursino G.R. et al. Long-term oral contraceptive use does not affect trabecular bone density // Am. J. Obstet. Gynecol. - 1989. - 160. - P. 402-404.

55. Lloyd T., Petit M.A., Lin H.M. et al. Lifestyle factors and the development of bone mass and bone strength in young women // J. Pediatr. - 2004. - 144. - P. 776-782.

56. Lloyd T., Taylor D.S., Lin H.M. et al. Oral contraceptive use by teenage women does not affect peak bone mass: a longitudinal study // Fertil. Steril. $-2000 .-74$. - P. 734-738.

57. Mais V., Fruzzetti F., Ajossa S. et al. Bone metabolism in young women taking a monophasic pill containing $20 \mathrm{mcg}$ ethinylestradiol: a prospective study // Contraception. - 1993. 48. - P. 445-452.

58. Masaryk P., Lunt M., Benevolenskaya L. et al. Effects of menstrual history and use of medications on bone mineral density: the EVOS Study // Calcif. Tissue Int. - 1998. - 63. P. 271-276

59. Mazess R.B., Barden H.S. Bone density in premenopausal women: effects of age, dietary intake, physical activity, smoking, and birth control pills // Am. J. Clin. Nutr. - 1991. 53. - P. 132-142.

60. Meyer N.L., Shaw J.M., Manore M.M. et al. Bone mineral density of Olympic-level female winter sport athletes // Med. Sci. Sports Exerc. - 2004. - 36. - P. 1594-1601.

61. Michaelsson K., Baron J.A., Farahmand B.Y. et al. Oral-contraceptive use and risk of hip fracture: a case-control study // Lancet. - 1999. - 353(9163). - P. 1481-1484.

62. Monteiro-Dantas C., Espejo-Arce X., Lui-Fil J.F. et al. A three-year longitudinal evaluation of the forearm bone density of users of etonogestrel- and levonorgestrel-releasing contraceptive implants // Reprod Health. - 2007. - 4. - P. 11-16.

63. Muñoz M.T., Morandé G., García-Centenera J.A. et al. The effects of estrogen administration on bone mineral density in adolescents with anorexia nervosa // Eur. J. Endocrinol. 2002. - 146. - P. 45-50

64. Murphy S., Khaw K.T., Compston J.E. Lack of relationship between hip and spine bone mineral density and oral contraceptive use // Eur. J. Clin. Invest. - 1993. - 23. - P. 108111. 
65. Naessen T., Olsson S.E., Gudmundson J. Differential effects on bone density of progestogen-only methods for contraception in premenopausal women // Contraception. - 1995. 52. - P. 35-39.

66. Nappi C., Di Spiezio Sardo A., Acunzo G. et al. Effects of a low-dose and ultra-low-dose combined oral contraceptive use on bone turnover and bone mineral density in young fertile women: a prospective controlled randomized study // Contraception. - 2003. - 67. - P. 355-359.

67. Nappi C., Di Spiezio Sardo A., Greco E. et al. Effects of an oral contraceptive containing drospirenone on bone turnover and bone mineral density // Obstet. Gynecol. - 2005. 105. - P. 53-60

68. Orr-Walker B.J., Evans M.C., Ames R.W. et al. The effect of past use of the injectable contraceptive depot medroxyprogesterone acetate on bone mineral density in normal postmenopausal women // Clin. Endocrinol (Oxf.). - 1998. 49. - P. 615-618.

69. Ott S.M., Scholes D., Lacroix A.Z. et al. Effects of oral contraceptive use on bone biochemical markers in young women // J. Clin. Endocrinol. Metab. - 2001. - 86. - P. 179-185.

70. Paoletti A.M., Orrù M., Floris S. et al. Evidence that treatment with monophasic oral contraceptive formulations containing ethinylestradiol plus gestodene reduces bone resorption in young women // Contraception. $-2000 .-61$. P. 259-263.

71. Paoletti A.M., Orru M., Lello S. et al. Short-term variations in bone remodeling markers of an oral contraception formulation containing $3 \mathrm{mg}$ of drospirenone plus 30 microg of ethinyl estradiol: observational study in young postadolescent women // Contraception. - 2004. - 70. - P. 293-298.

72. Pasco J.A., Kotowicz M.A., Henry M.J. et al. Oral contraceptives and bone mineral density: a population-based study // Am. J. Obstet. Gynecol. - 2000. - 182. - P. 265-269.

73. Perrotti M., Bahamondes L., Petta C. et al. Forearm bone density in long-term users of oral combined contraceptives and depot medroxyprogesterone acetate // Fertil. Steril. 2001. - 76. - P. 469-773.

74. Petitti D.B., Piaggio G., Mehta S. et al. Steroid hormone contraception and bone mineral density: a cross-sectional study in an international population // Obstet. Gynecol. - 2000. 95. - P. 736-744.

75. Polatti F., Perotti F., Filippa N. et al. Bone mass and long-term monophasic oral contraceptive treatment in young women // Contraception. - 1995. - 51. - 221 - 224 .

76. Prior J.C., Kirkland S.A., Joseph L. et al. Oral contraceptive use and bone mineral density in premenopausal women: cross-sectional, population-based data from the Canadian Multicentre Osteoporosis Study // CMAJ. - 2001. - 165. P. 1023-1029.

77. Recker R.R., Davies K.M., Hinders S.M. et al. Bone gain in young adult women // JAMA. $-1992 .-268 .-$ P. 2403 2408.

78. Reed S.D., Scholes D., LaCroix A.Z. et al. Longitudinal changes in bone density in relation to oral contraceptive use // Contraception. - 2003. - 68. - P. 177-182.

79. Rickenlund A., Carlstrom K., Ekblom B. et al. Effects of oral contraceptives on body composition and physical performance in female athletes // J. Clin. Endocrinol. Metab. 2004. - 89. - P. 4364-4370.

80. Rome E., Ziegler J., Secic M. et al. Bone biochemical markers in adolescent girls using either depot medroxyprogesterone acetate or an oral contraceptive // J. Pediatr. Adolesc. Gynecol. - 2004. - 17. - P. 373-377.

81. Ruffing J.A., Nieves J. W., Zion M. et al. The influence of lifestyle, menstrual function and oral contraceptive use on bone mass and size in female military cadets // Nutr Metab. 2007. - P. 4-17

82. Scholes D., Hubbard R.A., Ichikawa L.E. et al. Oral Contraceptive Use and Bone Density Change in Adolescent and Young Adult Women: A Prospective Study of Age, Hormone Dose, and Discontinuation // J. Clin. Endocrinol. Metab. 2011. - 96(9). - P. 1380-1387.
83. Scholes D., Ichikawa L., LaCroix A.Z. et al. Oral contraceptive use and bone density in adolescent and young adult women // Contraception. - 2010. - 81(1). - P. 35-42.

84. Seeman E., Szmukler G.I., Formica C. et al. Osteoporosis in anorexia nervosa: the influence of peak bone density, bone loss, oral contraceptive use, and exercise // J. Bone Miner. Res. $-1992 .-7$. - P. 1467-1474.

85. Shargil A.A. Hormone replacement therapy in perimenopausal women with a triphasic contraceptive compound: a three year prospective study // Int. J. Fertil. - 1985. - 30. - 15 .

86. Sowers M., Wallace R.B., Lenke J.H. Correlates of forearm bone mass among women during maximal bone mineralization // Prev. Med. - 1985. - 14. - P. 585-596.

87. Stevenson J.C., Lees B., Devenport M. et al. Determinants of bone density in normal women: risk factors for future osteoporosis // BMJ. - 1989. - 298. - P. 924-928

88. Sundgot-Borgen J., Torstveit M.K. Prevalence of eating disorders in elite athletes is higher than in the general population // Clin. J. Sport Med. - 2004. - 14. - P. 25-32.

89. Tuppurainen M., Kroger H., Saarikoski S. et al. The effect of previous oral contraceptive use on bone mineral density in perimenopausal women // Osteoporos. Int. - 1994. 4. - P. 93-98.

90. Ulrich C.M., Georgiou C.C., Snow-Harter C.M. et al. Bone mineral density in mother-daughter pairs: relations to lifetime exercise, lifetime milk consumption, and calcium supplements // Am. J. Clin. Nutr. - 1996. - 63. - P. 72-79.

91. Vessey M., Mant J., Painter R. Oral contraception and other factors in relation to hospital referral for fracture. Findings in a large cohort study // Contraception. - 1998. 57(4). - P. 231-235.

92. Vestergaard P., Rejnmark L., Mosekilde L. Oral contraceptive use and risk of fractures // Contraception. - 2006. 73(6). - P. 571-576.

93. Volpe A., Amram A., Cagnacci A. et al. Biochemical aspects of hormonal contraception: effects on bone mineral density and metabolism // Eur. J. Contracept. Reprod. Health Care. $-1997 .-2$. - P. 123-126.

94. Volpe A., Silferi M., Genazzani A.D. et al. Contraception in older women // Contraception. - 1993. - 47. - P. 229239.

95. Wallace L.S., Ballard J.E. Lifetime physical activity and calcium intake related to bone density in young women // J. Women Health Gend. Based Med. - 2002. - 11. - P. 389398.

96. Wanichsetakul P., Kamudhamar A., Watanarunagkaut $\mathrm{P}$. et al. Bone mineral density at various anatomic bone sites in women receiving combined oral contraceptives and depot medroxyprogesterone acetate for contraception // Contraception. - 2002. - 65. - P. 407-410.

97. Weaver C.M., Teegarden D., Lyle R.M. et al. Impact of exercise on bone health and contraindication of oral contraceptive use in young women women // Med. Sci. Sports Exerc. 2001. - 33. - P. 873-880.

98. WHO Statement on Hormonal Contraception and Bone Health, http://www.who.int/reproductivehealth/publications/ family planning/hc bone health/en/index.html, 2005.

99. WHO Statement on Hormonal Contraception and Bone Health, http://www.who.int/reproductivehealth/topics/family_planning/pbrief_bonehealth.pdf, 2007.

100. WHO. http://www.who.int/reproductivehealth/publications/family planning/9789241563888/en/ 4th. Geneva: WHO; 2009. Medical elegibility criteria for contraceptive use.

101. Wiegratz I., Thaler C.J. Hormonal Contraception What Kind, When, and for Whom? // Dtsch. Arztebl. Int. 2011. - 108(28-29). - P. 495-506.

102. Wilkins K., Johansen H., Beaudet M.P. et al. Oral contraceptive use // Health Rep. - 2001. - 11. - P. 25-37.

103. Zanker C.L., Cooke C.B., Truscott J.G. et al. Annual changes of bone density over 12 years in an amenorrheic athlete // Med. Sci. Sports Exerc. - 2004. - 36. - P. 137-142.

Получено 27.02.13 